\title{
Comparison of wear models for simulation of railway wheel polygonalization
}

\author{
Bo Peng ${ }^{1,2, *}$, Simon Iwnicki ${ }^{1}$, Philip Shackleton ${ }^{1}$, David Crosbee ${ }^{1}$ \\ ${ }^{1}$ Institute of Railway Research, University of Huddersfield, Huddersfield, UK \\ ${ }^{2}$ CRRC Zhuzhou Locomotive Co.,Ltd. Zhuzhou, China \\ ${ }^{*}$ E-mail: Bo.Peng@hud.ac.uk
}

\begin{abstract}
This paper presents a comparative study on the applicability of existing popular wear models in simulation of railway wheel polygonalization. Four wear models developed by BRR (British Railway Research), KTH (Royal Institute of Technology), USFD (University of Sheffield), and Professor Zobory respectively, are selected for the comparison, with consideration of global and local methods. All the wear models are firstly converted to calculate the instantaneous wear of one contact patch. A uniform expression of the converted wear functions is analytically derived, which allows a quantitative comparison between each wear model. Several scenarios grouped by simple excitation and complex excitation are adopted to assess the fluctuation of the instantaneous wear depth calculated by different wear models. The evolved polygonal wear around the wheel circumference is also compared among the wear models based on a developed prediction program. Simulation results show that all the wear models being investigated in this paper present a similar ability to reflect the fluctuation of the instantaneous wear under various circumstances. Specifically, the fluctuation of the instantaneous wear is obtained with almost the same results using different wear models with respect to the frequency and phase. While the mean value and the fluctuation amplitude of the instantaneous wear, as well as the roughness level of the evolved polygonal wear are all determined by the original wear coefficients used. The derived equivalent wear coefficient is a useful index to identify the proportional relationship among the wear models. Besides, the global method is believed not to be suitable for calculating the polygonal wear of railway wheels due to sharp corners probably generated by the absolute operation for the global WI (Wear Index).
\end{abstract}

Keywords: Wear models; railway wheel polygonization; instantaneous wear; comparison; simulation.

\section{Introduction}

The problem of railway wheel polygonalization has been a critical issue affecting the normal operation of the railway network along with the increase of vehicle speed and axle load. To investigate the mechanism of this phenomena through simulation, a prediction tool that can simulate the evolution of railway wheel polygonalization is necessary. In order to achieve such a prediction tool, an essential aspect is the selection and use of a wear model that should be suitable to simulate the circumferential wear of wheels. However, almost all of the existing wear models for rail/wheel application in literature were initially designed to predict the wheel transversal profile change for a long distance of running. When focusing on the prediction of wheel polygonalization, the wear models have to be converted to calculate the instantaneous wear of each contact patch for every time step in sequence, so that the wheel circumferential shape is allowed to evolve. In this sense, the converted wear model is actually no longer a "long-term" wear model, but an "instantaneous" wear model instead, although an amplification coefficient can be multiplied to the "instantaneous" wear to save computing time. This "instantaneous" wear model should be able to distinguish the dominant frequencies of the contact responses, so that a study on the frequency-fixed mechanism is possible. The fluctuation amplitude and phase of the instantaneous wear are also of interest, and these can depend 
on the characters of the converted "instantaneous" wear model. As a result, it is a fundamental issue to understand the applicability of existing wear models for the simulation of railway wheel polygonalization.

Several wear models have been developed for wheel-rail applications [1-8]. The main task of establishing a wear model is to find a quantitative relationship between the contact parameters and the material loss. The contact parameter inspected is the so-called WI (Wear Index) as an indicator for the wear calculation. The quantitative relationship is represented with wear coefficients that can only be obtained through experiments. Normally some wear regimes (e.g. mild, severe, and catastrophic) will be partitioned with corresponding wear coefficients depending on the level of WI. Interestingly, different wear models were developed with various WI, wear coefficients, and partition criteria of wear regimes as well, possibly due to different circumstances considered or different focus concentrated. Some researchers have carried out a comparative study on different wear models: Arizon [9] compared 4 wear functions, developed by Archard [10], Zobory [3], Pearce and Sherratt [2], and Jendel [5]/Enblom [11] respectively, by rewriting them in a common form with a wear velocity introduced as the uniform wear indicator. The quantitative relations between each wear model were obtained for mild and severe regime respectively. Pombo [12] compared 3 wear models developed by BRR, KTH, and USFD (named with the research group), with consideration of global and local methods. The comparison results revealed a good agreement in wear prediction obtained with the 3 wear models. Ding [13] carried out a comprehensive comparison on 4 wear models developed by Pearce [2], Zobory [3], Jendel [5] and Braghin [14]. The above mentioned comparative work were all standing in the perspective of wheel transversal profile prediction. Little research can be found from the perspective of wheel circumferential profile prediction. A typical one was from Johansson [15] who compared the Archard wear model and the frictional-work wear model in calculation of the wear distribution in his PhD thesis. It turned out that both methods lead to rather similar wear depth. Currently, the most widely used wear models for predicting railway wheel polygonalization are the Archard wear model which in its original form is feasible to calculate the instantaneous wear depth directly [16-18], as well as the frictional-work wear models which however need some conversions $[19,20]$. Among all the existing popular wear models available for wheel-rail applications, it is imperative to have a thorough understanding of their applicability in railway wheel polygonalization before they are adopted in the simulation program. This has not been investigated in a comprehensive sense, and is expected to be filled in this work.

In this paper, four representative wear models developed by BRR [2], KTH [5], USFD [6], and Professor Zobory [3] respectively, are selected to carry out a comparative study on their applicability in simulation of railway wheel polygonization. All the wear models are first converted to allow the calculation of instantaneous wear according to specific assumptions. A uniform expression of all the converted wear functions is analytically derived to facilitate the comparison between each wear model. Several scenarios grouped by simple excitation and complex excitation are adopted to assess the fluctuation of the instantaneous wear depth calculated by different wear models. The evolved polygonal wear around the wheel circumference is also compared among the wear models based on a developed program [17] for predicting railway wheel polygonalization.

\section{Wear models}

In this section, some assumptions are first stated for the calculation of instantaneous wear depth of one contact patch. Then after briefly introducing the original definition of the wear models, the equation conversions are illustrated in detail. Finally, a uniform expression of the converted wear functions is derived. 


\subsection{Assumptions}

- The wheel transversal profile is assumed not to change, with the focus only on the change in the wheel circumferential profile. This assumption is supported by the fact that, when railway wheel polygonalization occurs, the circumferential shapes measured at different wheel lateral positions are quite similar [21, 22].

- The instantaneous wear is assumed to be equally distributed in the axle direction, which is also consistent with the first assumption. For each contact patch, an averaged wear depth will be calculated.

- Only abrasive wear mechanism is accounted for, implying that the wear only occurs in the sliding zone of the contact patch. Hertz contact theory is adopted as the normal contact model, and FASTSIM [23] as the tangential contact model in this paper. Thus the contact area is an ellipse (with a: longitudinal semi-axis, and b: lateral semi-axis).

\subsection{Definition of Wear models}

Table 1 Wear models

\begin{tabular}{|c|c|c|c|c|c|c|}
\hline Wear models & Wear functions & \multicolumn{4}{|c|}{ Wear regimes } & Remarks \\
\hline \multirow{4}{*}{ BRR } & Wear rate $\left[\frac{m^{2}}{m}\right]$ & \multicolumn{4}{|c|}{ Wear index $T \gamma[N]$} & \multirow{4}{*}{$\begin{array}{l}\text { Area loss per } \\
\text { distance } \\
\text { rolled }\end{array}$} \\
\hline & $0.25 \frac{T \gamma}{D} \times 10^{-12}$ & \multicolumn{4}{|l|}{$T \gamma<100$} & \\
\hline & $\frac{25}{D} \times 10^{-12}$ & \multicolumn{4}{|c|}{$100 \leq T \gamma \leq 200$} & \\
\hline & $\frac{1.19 T \gamma-154}{D} \times 10^{-12}$ & \multicolumn{4}{|l|}{$T \gamma>200$} & \\
\hline \multirow{4}{*}{ KTH } & Wear volume $\left[\mathrm{m}^{3}\right]$ & $p_{z}[\mathrm{~Pa}]$ & & & $K_{K T H}[-]$ & \multirow{4}{*}{$\begin{array}{l}\text { Volume of } \\
\text { worn material }\end{array}$} \\
\hline & \multirow{3}{*}{$K_{K T H} \frac{N d}{H}$} & $p_{z}<0.8 H$ & \multicolumn{2}{|c|}{$s<0.2$ or $s>0.7$} & $1 \square 10 \times 10^{-4}$ & \\
\hline & & $p_{z}<0.8 H$ & \multicolumn{2}{|c|}{$0.2 \leq s \leq 0.7$} & $30 \square 40 \times 10^{-4}$ & \\
\hline & & $p_{z}>0.8 H$ & \multicolumn{2}{|c|}{ any $s$} & $300 \square 400 \times 10^{-4}$ & \\
\hline \multirow{4}{*}{ USFD } & Wear rate $\left[\frac{k g}{m \cdot m^{2}}\right]$ & \multicolumn{4}{|c|}{ Wear index $\frac{T \gamma}{A}\left[\mathrm{~N} / \mathrm{m}^{2}\right]$} & \multirow{4}{*}{$\begin{array}{l}\text { Mass loss per } \\
\text { running } \\
\text { distance per } \\
\text { contact area }\end{array}$} \\
\hline & $5.3 \frac{T \gamma}{A} \times 10^{-9}$ & \multicolumn{4}{|c|}{$\frac{T \gamma}{A}<10.4 \times 10^{6}$} & \\
\hline & $55.0 \times 10^{-9}$ & \multicolumn{4}{|c|}{$10.4 \times 10^{6}<\frac{T \gamma}{A}<77.2 \times 10^{6}$} & \\
\hline & $61.9 \frac{T \gamma}{A} \times 10^{-9}$ & \multicolumn{4}{|c|}{$\frac{T \gamma}{A}>77.2 \times 10^{6}$} & \\
\hline \multirow{3}{*}{ Zobory } & Wear rate $\left[\frac{k g}{s \cdot m^{2}}\right]$ & \multicolumn{2}{|c|}{$\dot{E}\left[\mathrm{~N} \cdot \mathrm{m} / \mathrm{s} \cdot \mathrm{m}^{2}\right]$} & \multicolumn{2}{|c|}{$K_{\text {Zobory }}\left[\frac{k g}{N \cdot m}\right]$} & \multirow{3}{*}{$\begin{array}{l}\text { Stochastic } \\
\text { energy flow } \\
\text { density }\end{array}$} \\
\hline & \multirow{2}{*}{$K_{\text {Zobory }} \dot{E}$} & \multicolumn{2}{|c|}{$\dot{E}<4 \times 10^{-6}$} & \multicolumn{2}{|c|}{ around $7 \times 10^{-10}$} & \\
\hline & & \multicolumn{2}{|l|}{$\dot{E}>4 \times 10^{-6}$} & \multicolumn{2}{|c|}{ around $21 \times 10^{-10}$} & \\
\hline
\end{tabular}


The original definitions of the four wear models being investigated are listed in Table 1. To facilitate the comparison, all the original wear functions are converted to SI units. As can be seen in Table 1, these wear models in their original definitions have different equation forms with different WI as the wear indicators (the variables in the equation). The partition criteria of the wear regimes is only applicable to the respective weal model on its own, and thus not comparable between different wear models. The wear coefficients are also varied due to different experimental scenarios and different materials tested.

\subsection{Conversion of Wear models}

Define:

$$
T \gamma=\left|T_{x} \gamma_{x}\right|+\left|T_{y} \gamma_{y}\right|+|M \phi|
$$

Define:

$$
\tau(x, y) \xi(x, y)=\sqrt{\tau_{x}^{2}(x, y)+\tau_{y}^{2}(x, y)} \cdot \sqrt{\xi_{x}^{2}(x, y)+\xi_{y}^{2}(x, y)}
$$

Where $T_{x}, T_{y}$, and $M$ are the longitudinal tangential force, the lateral tangential force, and the spin torque respectively; $\gamma_{x}, \gamma_{y}$, and $\phi$ are the global longitudinal creepage, the global lateral creepage, and the spin respectively; $\tau(x, y)$ and $\xi(x, y)$ are the tangential stress distribution and resultant creepage (local) in contact grid respectively, with $(\mathrm{x}, \mathrm{y})$ indicating the local coordinates in the contact grid, and with subscript $\mathrm{x}$ and $\mathrm{y}$ indicating the direction components. $T \gamma$ will be used as the WI for global methods, and $\tau(x, y) \xi(x, y)$ for local methods.

Without losing generality, define:

$$
\begin{gathered}
W_{B R R}=K_{B R R} \cdot \frac{T \gamma}{D}\left[\frac{\mathrm{m}^{2}}{\mathrm{~m}}\right] \\
W_{U S F D}=K_{U S F D} \cdot \frac{T \gamma}{A}\left[\frac{\mathrm{kg}}{\mathrm{m} \cdot \mathrm{m}^{2}}\right] \\
W_{\text {Zobory }}=K_{\text {Zobory }} \cdot \dot{E}\left[\frac{\mathrm{kg}}{\mathrm{s} \cdot \mathrm{m}^{2}}\right]
\end{gathered}
$$

Where: $W$ is the wear rate and $K$ the wear coefficient, both with subscript indicating the wear model.

Note that the KTH wear model is not defined as a wear rate, but a specific value of worn volume for given sliding distance and normal force. Obviously, it can be directly used to calculate the instantaneous wear. For those wear models defined as a wear rate, a "running distance" over one contact patch must be correctly multiplied to the wear rate to obtain the instantaneous wear. The obtained wear could be in the form of area, volume, or mass, depending on the wear models' definition. By averaging the instantaneous wear of the contact patch, a single-value wear depth is obtained. For the global method, the averaging method is different depending on the wear models' definition. For the local method, the wear distribution of one contact patch is averaged by accumulating the wear distribution longitudinally and then averaging laterally.

\subsubsection{BRR model}

The BRR wear model can only be used globally. According to the definition of the BRR wear model, to calculate the instantaneous wear for one contact patch, the "running distance" should be one wheel revolution which is 
associated with the wheel diameter. The calculated BRR wear is the area loss along the wheel transversal profile. Then the area loss is divided by $2 b$ to obtain the averaged wear depth. The conversion is given in Equation (6). As can be seen, the wheel diameter is actually counteracted.

$$
H_{B R R}=W_{B R R} \cdot(\pi D) \cdot \frac{1}{2 b}=K_{B R R} \frac{T \gamma}{D} \cdot(\pi D) \cdot \frac{1}{2 b}=\frac{\pi K_{B R R}}{2 b} \cdot T \gamma
$$

If assuming $K_{B R R}^{*}=\frac{\pi K_{B R R}}{2 b}$, Equation (6) becomes:

$$
H_{B R R}=K_{B R R}^{*} \cdot T \gamma
$$

\subsubsection{KTH model}

The KTH wear model was originally developed only for the local method [5] based on the Archard wear model [10]. The basic equation is as below:

$$
V_{\text {wear }}=K_{K T H} \frac{N d}{\mathrm{H}}
$$

Where: $V_{\text {wear }}$ is the worn volume, $N$ the normal force, $d$ the sliding distance, and $\mathrm{H}$ the material hardness.

The corresponding form for calculating the wear distribution can be written as:

$$
h_{K T H}(x, y)=K_{K T H} \frac{p_{z}(x, y) \cdot \Delta d(x, y)}{\mathrm{H}}
$$

Where: $h_{K T H}(x, y)$ is the wear depth distribution, $p_{z}(x, y)$ the normal pressure distribution, and $\Delta d(x, y)$ the local sliding distance distribution. Note that the wear only occurs in the sliding zone where the traction bound is saturated and where the tangential stress is exactly proportional to the normal pressure with the friction coefficient $\mu: \tau(x, y)=\mu p_{z}(x, y)$ where $(x, y) \in$ sliding zone . Together with $\Delta d(x, y)=0$ where $(x, y) \notin$ sliding zone , $p_{z}(x, y) \cdot \Delta d(x, y)$ can be decomposed into:

$$
p_{z}(x, y) \cdot \Delta d(x, y)= \begin{cases}\frac{\tau(x, y)}{\mu} \Delta d(x, y) & (x, y) \in \text { sliding zone } \\ 0 & (x, y) \notin \text { sliding zone }\end{cases}
$$

Actually, as $\Delta d(x, y)$ can determine the sliding/adhesion zone on its own, Equation (10) can be recombined to a concise expression as below:

$$
p_{z}(x, y) \cdot \Delta d(x, y)=\frac{\tau(x, y)}{\mu} \Delta d(x, y)
$$

In addition, $\Delta d(x, y)$ can be further transformed through the following equation:

$$
\Delta d(x, y)=s(x, y) \cdot \Delta t=V \cdot \xi(x, y) \cdot \frac{\Delta x}{V}=\xi(x, y) \cdot \Delta x
$$

Where: $s(x, y)$ is the resultant sliding velocity in grid element, $\Delta t$ the time passing through one grid element, $\Delta x$ the longitudinal length of one grid element, and $V$ the vehicle speed. Here $\Delta t \approx \Delta x / V$ is assumed. By substituting Equation (11) and (12) to Equation (9), it becomes: 


$$
h_{K T H}(x, y)=\frac{K_{K T H} \cdot \Delta x}{\mu \mathrm{H}} \cdot \tau(x, y) \xi(x, y)
$$

Given $\Delta x=2 a / m$, where $m$ is the longitudinal meshing number, Equation (13) becomes:

$$
h_{K T H}(x, y)=\frac{2 a \cdot K_{K T H}}{m \mu \mathrm{H}} \cdot \tau(x, y) \xi(x, y)
$$

If assuming $K_{K T H}^{\#}=\frac{2 a K_{K T H}}{m \mu H}$, Equation (14) becomes:

$$
h_{K T H}(x, y)=K_{K T H}^{\#} \cdot \tau(x, y) \xi(x, y)
$$

The KTH wear model is based on the Archard wear model. If the Archard wear model is used globally, the key problem is that there is no way for the spin to be accounted for in the calculation of global sliding distance. Therefore, it is only feasible for cases of small spin, which will definitely limit the application of Archard wear model in the global method. As a result, this method is not included in this paper.

\subsubsection{USFD model}

The USFD wear model can be used both globally and locally. For the global method, the "running distance" over one contact patch should be the longitudinal contact patch length $(2 a)$. Correspondingly for the local method, the "running distance" over one grid element is the longitudinal element length $(\Delta x)$. It is worth mentioning that the "running distance" here is different from that of the BRR wear model which is the wheel perimeter. This is due to the different definition of the wear functions. The calculated USFD wear is in the form of mass loss. By further dividing the mass loss by the wheel density, the averaged wear depth of the contact patch is obtained.

Note that, for global method the WI is $T \gamma / A$, while for local method the WI is $\tau(x, y) \xi(x, y)$. The derivation of the WI for local method can refer to [14]. The wear rate in the global and local methods is rewritten as below respectively:

$$
\begin{gathered}
W_{U S F D}=K_{U S F D} \frac{T \gamma}{A} \\
W_{U S F D}(x, y)=K_{U S F D} \cdot \tau(x, y) \xi(x, y)
\end{gathered}
$$

The conversions of USFD wear model in global and local methods are given below respectively:

$$
\begin{gathered}
H_{U S F D}=W_{U S F D} \cdot \frac{2 a}{\rho}=K_{U S F D} \frac{T \gamma}{\pi a b} \cdot \frac{2 a}{\rho}=\frac{2 K_{U S F D}}{\pi b \rho} \cdot T \gamma \\
h_{U S F D}(x, y)=W_{U S F D}(x, y) \cdot \frac{\Delta x}{\rho}=K_{U S F D} \cdot \tau(x, y) \xi(x, y) \cdot \frac{\Delta x}{\rho}=\frac{K_{U S F D} \cdot \Delta x}{\rho} \cdot \tau(x, y) \xi(x, y)
\end{gathered}
$$

Given $\Delta x=2 a / m$, Equation (19) becomes:

$$
h_{U S F D}(x, y)==\frac{2 a K_{U S F D}}{m \rho} \cdot \tau(x, y) \xi(x, y)
$$

If assuming $K_{U S F D}^{*}=\frac{2 K_{U S F D}}{\pi b \rho}$ and $K_{U S F D}^{\#}=\frac{2 a K_{U S F D}}{m \rho}$, Equation (18) and Equation (20) become: 


$$
\begin{gathered}
H_{U S F D}=K_{U S F D}^{*} \cdot T \gamma \\
h_{U S F D}(x, y)=K_{U S F D}^{\#} \cdot \tau(x, y) \xi(x, y)
\end{gathered}
$$

\subsubsection{Zobory model}

The Zobory wear model was basically proposed for local method. Professor Zobory introduced $\dot{E}$ (stochastic energy flow density) as the WI, which is expressed as below:

$$
\dot{E}(x, y)=\tau(x, y) s(x, y)
$$

To save the calculation time, Profess Zobory also proposed a simplified method, which is illustrated as below:

$$
\dot{E}=\frac{\left|T_{x} v_{x}\right|+\left|T_{y} v_{y}\right|+|M \omega|}{A} \cdot \frac{p_{z}(x, y)}{\mathrm{P}_{\mathrm{z}}}
$$

Where: $v_{x}, v_{y}$, and $\omega$ are the longitudinal sliding velocity (global), the lateral sliding velocity (global), and the angular sliding velocity, respectively; $\mathrm{P}_{\mathrm{z}}$ is the mean normal pressure.

The simplification is to assume that the wear is distributed proportionally to the normal pressure by introducing the term $p_{z}(x, y) / \mathrm{P}_{\mathrm{z}}$ to Equation (24). However, based on the assumption of this paper, the wear distribution will eventually be averaged to get a single-value wear depth. Under this circumstance, the term $p_{z}(x, y) / \mathrm{P}_{\mathrm{z}}$ is actually not useful for the final result and thus can be just excluded from the equation. Thus a global approach based on the simplified method above can be proposed by rewriting Equation (24) as below:

$$
\dot{E}=\frac{\left|T_{x} v_{x}\right|+\left|T_{y} v_{y}\right|+|M \omega|}{A}
$$

To convert the Zobory wear model to calculate the instantaneous wear, a "running time" instead of a "running distance" is to be multiplied to the wear rate, because the WI of the Zobory wear model $(\dot{E})$ is a velocity-related term. The "running time" passing through one contact patch should be $d t=2 a / V$ for global method and $\Delta t=\Delta x / V$ for local method, respectively. The calculated wear is in the form of mass loss. By further dividing the mass loss with the wheel density, the averaged wear depth of the contact patch is obtained.

The wear rate of Zobory wear model for global and local methods are derived as below respectively:

$$
\begin{gathered}
W_{\text {Zobory }}=K_{\text {Zobory }} \cdot \dot{E}=K_{\text {Zobory }} \frac{\left|T_{x} v_{x}\right|+\left|T_{y} v_{y}\right|+|M \omega|}{A}=K_{\text {Zobory }} \cdot V \cdot \frac{\left|T_{x} \gamma_{x}\right|+\left|T_{y} \gamma_{y}\right|+|M \phi|}{A}=K_{\text {Zobory }} \frac{V \cdot T \gamma}{A} \\
W_{\text {Zobory }}(x, y)=K_{\text {Zobory }} \cdot \dot{E}(x, y)=K_{\text {Zobory }} \cdot \tau(x, y) s(x, y)=K_{\text {Zobory }} \cdot V \cdot \tau(x, y) \xi(x, y)
\end{gathered}
$$

The conversions of Zobory wear model in global and local methods are given below, respectively.

$$
\begin{gathered}
H_{\text {Zobory }}=W_{\text {Zobory }} \cdot \frac{d t}{\rho}=K_{\text {Zobory }} \frac{V \cdot T \gamma}{\pi a b} \cdot \frac{2 a}{V} \cdot \frac{1}{\rho}=\frac{2 K_{\text {Zobory }}}{\pi b \rho} \cdot T \gamma \\
h_{\text {Zobory }}(x, y)=W_{\text {Zobory }}(x, y) \cdot \frac{\Delta t}{\rho}=K_{\text {Zobory }} \cdot V \cdot \tau(x, y) \xi(x, y) \cdot \frac{\Delta x}{V} \cdot \frac{1}{\rho}=K_{\text {Zobory }} \cdot \tau(x, y) \xi(x, y) \cdot \frac{\Delta x}{\rho}
\end{gathered}
$$


Given $\Delta x=2 a / m$, Equation (29) becomes:

$$
h_{\text {Zobory }}(x, y)=\frac{2 a K_{\text {Zobory }}}{m \rho} \cdot \tau(x, y) \xi(x, y)
$$

If assuming $K_{\text {Zobory }}^{*}=\frac{2 K_{\text {Zobory }}}{\pi b \rho}$ and $K_{\text {Zobory }}^{\#}=\frac{2 a K_{\text {Zobory }}}{m \rho}$, Equation (28) and Equation (30) become:

$$
\begin{gathered}
H_{\text {Zobory }}=K_{\text {Zobory }}^{*} \cdot T \gamma \\
h_{\text {Zobory }}(x, y)=K_{\text {Zobory }}^{\#} \cdot \tau(x, y) \xi(x, y)
\end{gathered}
$$

As can be seen from the above derivation, the vehicle speed in the original WI is effectively counteracted. By comparing Equation (28) with Equation (18), and Equation (30) with Equation (20) respectively, it can be found that the converted equations of Zobory wear model and USFD wear model share an identical form, with the only difference being the original wear coefficient of each model. Consequently, it is believed that USFD wear model and Zobory wear model possess the same nature despite the different original WI used.

\begin{tabular}{|c|c|c|c|}
\hline \multirow{4}{*}{ 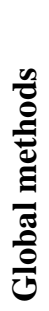 } & Uniform expression & $H=K^{*} \cdot T \gamma$ & Equivalent Coefficient $K^{*}[\mathrm{~m} / \mathrm{N}]$ \\
\hline & BRR & $H_{B R R}=K_{B R R}^{*} \cdot T \gamma$ & $K_{B R R}^{*}=\pi K_{B R R} / 2 b$ \\
\hline & USFD & $H_{U S F D}=K_{U S F D}^{*} \cdot T \gamma$ & $K_{U S F D}^{*}=2 K_{U S F D} / \pi b \rho$ \\
\hline & Zobory & $H_{\text {Zobory }}=K_{\text {Zobory }}^{*} \cdot T \gamma$ & $K_{\text {Zobory }}^{*}=2 K_{\text {Zobory }} / \pi b \rho$ \\
\hline \multirow{5}{*}{ 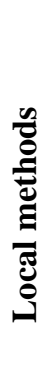 } & Uniform expression & $h(x, y)=K^{\#} \cdot \tau(x, y) \xi(x, y)$ & Equivalent Coefficient $K^{\#}\left[\mathrm{~m}^{3} / \mathrm{N}\right]$ \\
\hline & KTH & $h_{K T H}(x, y)=K_{K T H}^{\#} \cdot \tau(x, y) \xi(x, y)$ & $K_{K T H}^{\#}=2 a K_{K T H} / m \mu \mathrm{H}$ \\
\hline & USFD & $h_{U S F D}(x, y)=K_{U S F D}^{\#} \cdot \tau(x, y) \xi(x, y)$ & $K_{U S F D}^{\#}=2 a K_{U S F D} / m \rho$ \\
\hline & Zobory & $h_{\text {Zobory }}(x, y)=K_{\text {Zobory }}^{\#} \cdot \tau(x, y) \xi(x, y)$ & $K_{\text {Zobory }}^{\#}=2 a K_{\text {Zobory }} / m \rho$ \\
\hline & \multicolumn{3}{|c|}{ Handling wear distribution: Accumulate longitudinally and then average laterally } \\
\hline
\end{tabular}

\subsection{Uniform expression of converted wear models}

Table 2. The uniform expression of wear models converted for calculation of instantaneous wear depth

As a summary, all the converted equations grouped by global and local methods are listed in Table 2. It is interesting to see that, although the wear models in comparison are varied in their original expressions, all of them can be analytically transformed to a uniform expression. The uniform expression is $H=K^{*} \cdot T \gamma$ for global methods and $h(x, y)=K^{\#} \cdot \tau(x, y) \xi(x, y)$ for local methods. The uniform WI (variable part) is $T \gamma$ for global methods and $\tau(x, y) \xi(x, y)$ for local methods. An equivalent coefficient is derived for every wear model to represent the constant part in the uniform expression, which is denoted by $K^{*}$ for global methods and $K^{\#}$ for local methods, with subscripts indicating the wear models. The unit of the equivalent coefficient is also uniform, which is $\mathrm{m} / \mathrm{N}$ for $K^{*}$ and $\mathrm{m}^{3} / \mathrm{N}$ for $K^{\#}$. It has to be mentioned that, although a and b are actually variables in time history, the contribution of their variation to the variation of wear depth is negligible compared with $T \gamma$ or $\tau(x, y) \xi(x, y)$ 
, so that $\mathrm{a}$ and $\mathrm{b}$ are also deemed as constants to be included in the formula of equivalent coefficients, which makes the uniform expression more concise.

\section{Comparison of wear models}

The significance of Table 2 lies in that it allows a quantitative comparison among the wear models in the same group (either global methods or local methods). The proportional relation between each wear model can be obtained by simply comparing the equivalent coefficients (constant part) between each wear model, as the WI (variable part) is the same for all the wear models in the same group (global or local). Given a specific case and the original wear coefficients, the equivalent coefficients can be calculated to identify the ratio between each wear model. An example is presented in Table 3.

Table 3 An example for calculating the converted equivalent coefficients and instantaneous wear depths

\begin{tabular}{|c|c|c|c|c|c|c|c|c|}
\hline & Parameters & $\mathrm{Va}$ & & & Parameters & & Value & \\
\hline & $N$ & & {$[\mathrm{kN}]$} & & $K_{B R R}$ & & $0.25 \times 10^{-1}$ & {$\left[\mathrm{~m}^{2} / \mathrm{N}\right]$} \\
\hline & $\gamma_{x}$ & & $10^{-3}[-]$ & & $K_{K T H}$ & & $5 \times 10^{-4}$ & \\
\hline & $\gamma_{y}$ & & $10^{-3}[-]$ & & $K_{U S F D}$ & & $5.3 \times 10^{-9}$ & $\mathrm{~g} / \mathrm{Nm}]$ \\
\hline Inputs & $\phi$ & & {$[1 / \mathrm{m}]$} & & $K_{\text {Zobory }}$ & & $7 \times 10^{-10}$ & / $/ \mathrm{Nm}]$ \\
\hline & $a$ & & $86[\mathrm{~m}]$ & & $V$ & & $50[\mathrm{~km} / \mathrm{h}]$ & \\
\hline & $b$ & & $66[\mathrm{~m}]$ & & $\mu$ & & $.3[-]$ & \\
\hline & $\rho$ & & {$\left[\mathrm{kg} / \mathrm{m}^{3}\right]$} & & $\mathrm{H}$ & & $2.94[\mathrm{Gpa}$ & \\
\hline & & & & & $m$ & & $51[-]$ & \\
\hline & Global equi & alent coe & cients $[\mathrm{n}$ & & Local equi & ent coeft & icients $\left[\mathrm{m}^{3}\right.$ & \\
\hline Equivalent & $K^{*}$ & $K_{B R R}^{*}$ & $K_{U S F D}^{*}$ & $K_{\text {Zobory }}^{*}$ & $K^{\#}$ & $K_{K T H}^{\#}$ & $K_{U S F D}^{\#}$ & $K_{\text {Zobory }}^{\#}$ \\
\hline coefticients & Value & $4.1 \mathrm{e}-11$ & $4.5 \mathrm{e}-11$ & $5.9 \mathrm{e}-12$ & Value & $1.9 \mathrm{e}-16$ & $2.3 e-16$ & $3.0 \mathrm{e}-17$ \\
\hline & Ratio & 6.9 & 7.6 & 1 (ref) & Ratio & 6.4 & 7.6 & 1 (ref) \\
\hline & & Global & nethods & & & Local m & ethods & \\
\hline Wear denth & Model & $\overline{B R R}$ & USFD & Zobory & Model & $\mathrm{KTH}$ & USFD & Zobory \\
\hline & Value $[\mathrm{m}]$ & $2.4 \mathrm{e}-9$ & $2.6 e-9$ & $3.4 \mathrm{e}-10$ & Value $[\mathrm{m}]$ & $1.6 \mathrm{e}-9$ & $1.9 \mathrm{e}-9$ & $2.5 \mathrm{e}-9$ \\
\hline & Ratio [-] & 6.9 & 7.6 & 1 (ref) & Ratio [-] & 6.4 & 7.6 & 1 (ref) \\
\hline
\end{tabular}

* The Zobory wear model locally is the reference for the ratio calculation

As can be seen from Table 3, the ratio of the wear depths between each wear model is exactly the same with that of the equivalent coefficients. As a result, the equivalent coefficient can be used as a useful index to quantitatively compare the wear models between each other. But the quantitative comparison is basically limited in the same group (either global or local). As for the comparison of the same wear model between global and local methods, it is unlikely to obtain a quantitative relationship due to the complexity of the contact models. The discrepancy between global and local methods for the same wear model could depend on many parameters and is complicated to explain. In spite of this, it is found that the wear depth calculated by the global method is larger than that by the local method, and their ratio is convergent with the increase of grid density. A convergence analysis of the grid density on the discrepancy of instantaneous wear depth between global and local methods is presented in Figure 
1, with the parameters listed in Table 3. Here the USFD wear model is used as an example, the discrepancy between the instantaneous wear depth calculated by global and local methods is indicated with their ratio, and the element number is the same for both longitudinal and lateral direction. As can be seen from Figure 1, the element number should be big enough to get a convergent discrepancy between global and local methods for the same wear model. Considering the compromise of computing time, a minimum value of 50 for the element number is suggested.

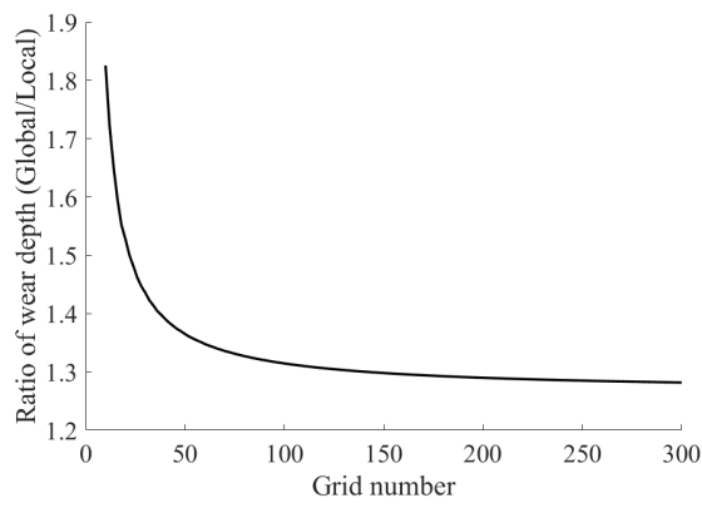

Figure 1 A convergence analysis of the element number on the ratio of wear depth (Global/Local): the USFD wear model is used in this analysis

Based on the above knowledge, further comparative studies can be carried out to look at how these converted "instantaneous" wear models reflect the fluctuation of the circumferential instantaneous wear under different circumstances, and whether they behave in the same way or not. A Chinese high-axle load locomotive is used as the research object for the following comparative studies. This locomotive is configured with 2 bogies, each of which consists of 2 wheelsets. The instantaneous wear depth is only calculated for the left wheel of the first wheelset. The track flexibility is simplified by positioning a sleeper underneath every wheelset. Only tangent track on which the wheel tread wear mainly occurs is taken into consideration, as the wheel polygonalization is merely formed in the wheel tread without contribution from the wheel flange wear occurring on curves. Typical original wear coefficients listed in Table 3 for a mild wear regime occurring on a tangent track are thus adopted. The vehicle speed is set to be $70 \mathrm{~km} / \mathrm{h}$ for all scenarios. The maximum frequency of interest is $300 \mathrm{~Hz}$ for a low-speed locomotive. Several scenarios grouped by simple excitation and complex excitation are investigated to observe the response feature of the wear models under different types of excitation. For every scenario, a comprehensive comparison among the wear models is implemented by inspecting the fluctuation of the instantaneous wear depths in 3 aspects: fluctuation frequency, fluctuation amplitude, and fluctuation phase.

\subsection{Under harmonic excitation}

To obtain a basic understanding, two simple harmonic cases are first studied: under excitation of vertical sinusoidal track irregularity and sinusoidal initial OOR, respectively. To facilitate the comparison, these two types of sinusoidal excitations are configured with the same parameters: wavelength of $1 / 10$ wheel circumference $(0.3925$ $\mathrm{m}$ ) and amplitude of $0.1 \mathrm{~mm}$. The only excitation frequency is around $50 \mathrm{~Hz}$ at speed of $70 \mathrm{~km} / \mathrm{h}$. This frequency is expected to be reflected by the wear model in the time history of the instantaneous wear depth. The simulation results are shown in Figure 2 and Figure 3 respectively, with 3 sub graphs showing the time history, the frequency spectrum, and the zoomed details demonstrating the fluctuation amplitude and phase. 


\subsubsection{Vertical sinusoidal track irregularity}

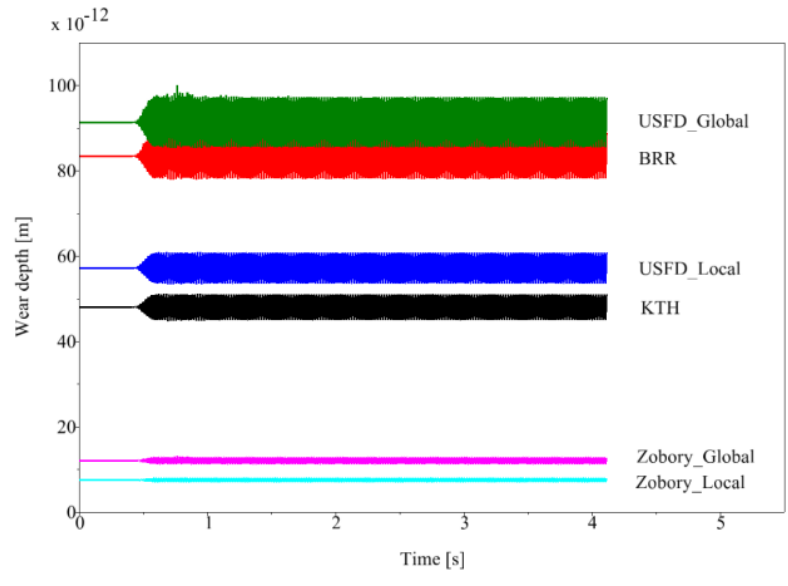

(a) Time history

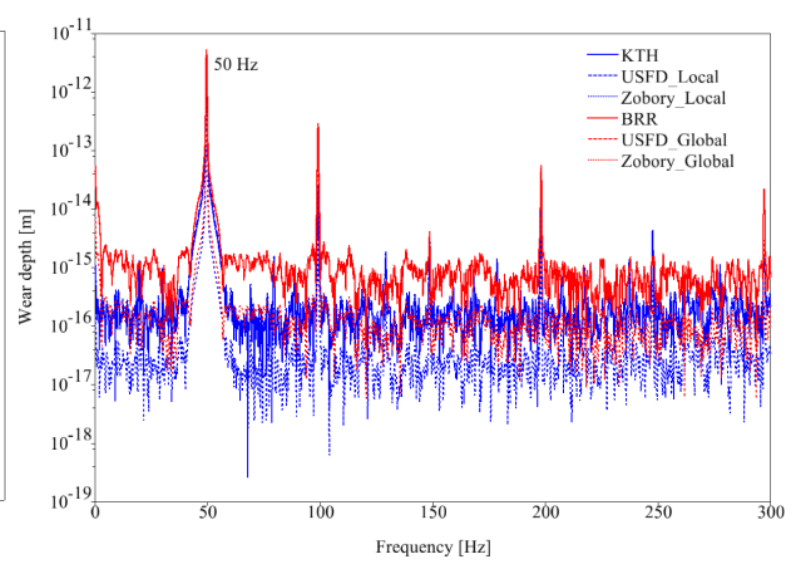

(b) Frequency spectrum

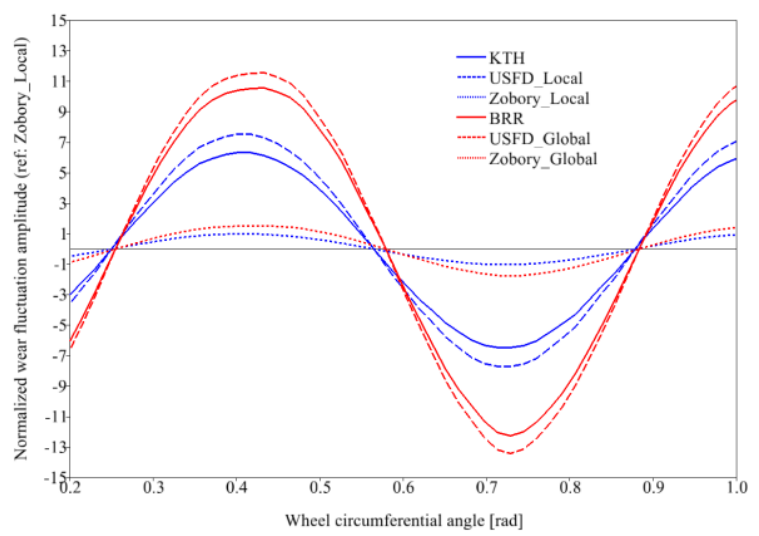

(c) Wear fluctuation phase and normalized wear fluctuation amplitude (ref: Zobory_Local)

Figure 2 Comparison of instantaneous wear depths among the wear models under excitation of a sinusoidal vertical track irregularity (wavelength: 1/10 wheel circumference, speed: 70km/h): (a) Time history, (b) Frequency spectrum, (c) Wear fluctuation phase and normalized wear fluctuation amplitude (ref: Zobory_Local)

As can be seen from Figure 2 (a), the time history of the instantaneous wear depths calculated by different wear models are presented in a comparable sense. The difference in the mean value of the instantaneous wear depth between each wear model is mainly determined by the original wear coefficients of each wear model, which has been discussed previously. A more interesting aspect in this paper is the fluctuation of the instantaneous wear depth. It can be seen from Figure 2 (b) that, all the wear models can correctly reflect the excitation frequency which is $50 \mathrm{~Hz}$ in this case. Figure 2 (c) further shows the amplitude and phase of the fluctuation of the instantaneous wear depths with the mean values subtracted. To make the wear fluctuation phase observable, the abscissa is zoomed in one period of fluctuations with the wheel circumferential angle as the x-coordinate. To compare the wear fluctuation amplitude conveniently, the y-coordinate is replaced by the normalized amplitudes of the wear fluctuations, where the Zobory_Local wear model with the smallest wear fluctuation is the reference and has a normalized fluctuation amplitude of 1 . As can be seen in Figure 2 (c), the wear fluctuation phase is exactly the same for the same group (either global or local wear models), only with a very small difference between global and local groups. In terms of the wear fluctuation amplitude, the ratio of the fluctuation amplitude among all the wear models is almost the same as that of the mean value. This can be checked in the following Table 4 . 
Table 4 A comparison between the ratio of the mean value and the ratio of fluctuation amplitude

\begin{tabular}{llllllll}
\hline \multirow{2}{*}{ Model } & BRR & $\begin{array}{l}\text { USFD } \\
\text { Global }\end{array}$ & $\begin{array}{l}\text { Zobory } \\
\text { Global }\end{array}$ & KTH & $\begin{array}{l}\text { USFD } \\
\text { Local }\end{array}$ & $\begin{array}{l}\text { Zobory } \\
\text { Local }\end{array}$ \\
\hline \multirow{2}{*}{ Ratio } & Mean value & 11.04 & 12.1 & 1.6 & 6.36 & 7.58 & 1 \\
& Fluctuation & 10.6 & 11.61 & 1.53 & 6.36 & 7.58 & 1 \\
\hline
\end{tabular}

* The Zobory wear model locally is the reference for the ratio calculation

\subsubsection{Sinusoidal initial OOR}

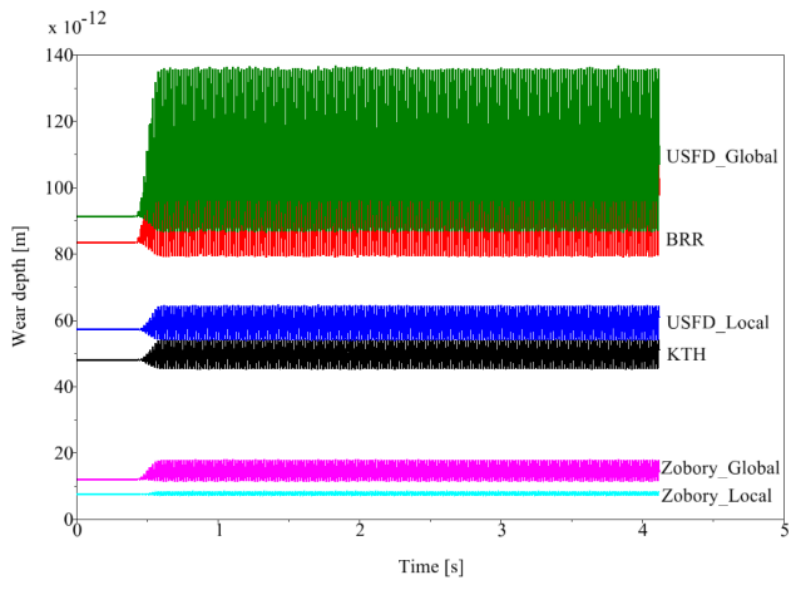

(a) Time history

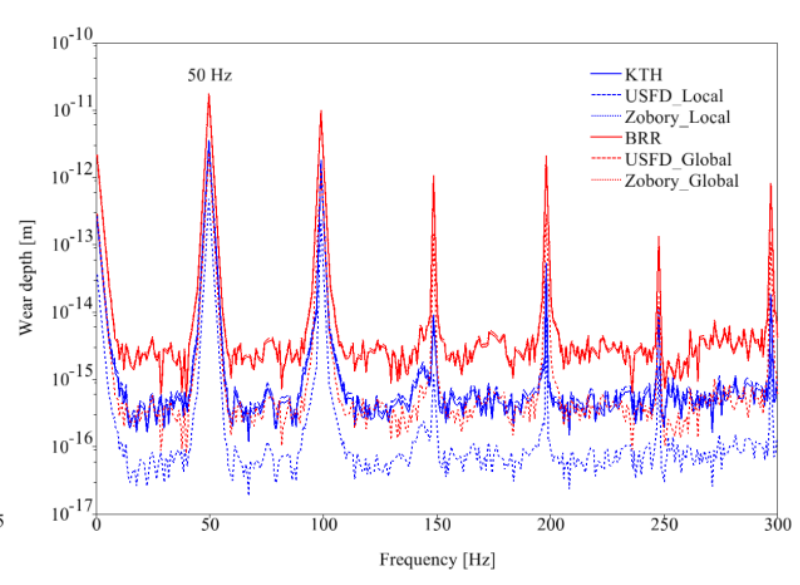

(b) Frequency spectrum

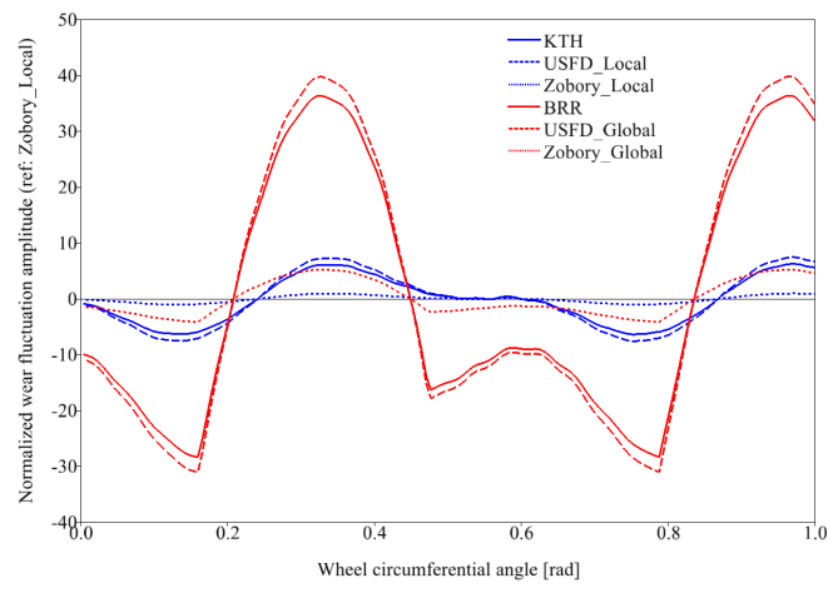

(c) Wear fluctuation phase and normalized wear fluctuation amplitude (ref: Zobory_Local)

Figure 3 Comparison of instantaneous wear depths among the wear models under excitation of a sinusoidal initial OOR (wavelength: 1/10 wheel circumference, speed: 70km/h): (a) Time history, (b) Frequency spectrum, (c) Wear fluctuation phase and normalized wear fluctuation amplitude (ref: Zobory_Local)

Figure 3 shows the results excited by a sinusoidal initial OOR. The basic feature is similar compared to the case of track irregularity. The excitation frequency can also be reflected correctly by all the wear models. But the multiple frequency components are more notable due to the cyclic revolution of the wheels, as showed in Figure 3 (b). In addition, some unreasonable sharp corners are found in the curves of global methods in Figure 3 (c). This is because of the simplification of global methods with the absolute operation for each component of $T \gamma$, which can be explained by the following. 
The WI of global methods is $T \gamma$ with 3 absolute components representing the wear works in 3 coordinates, which is rewritten as below. Note that an absolute operation is required for each component as negative frictional work is meaningless.

$$
T \gamma=\left|T_{x} \gamma_{x}\right|+\left|T_{y} \gamma_{y}\right|+|M \phi|
$$

Under an excitation with a single frequency, all the contact responses must share the same frequency component. If viewing every contact response as a sinusoidal curve mathematically, each component of $T \gamma$ comes down to a product of two sinusoidal curves with the same frequency. Note that any single sinusoidal curves can be expressed as a universal formula below:

$$
f(t)=\overline{\mathrm{M}}+\mathrm{A} \cdot \sin (\omega t+\theta)
$$

Where $\overline{\mathrm{M}}$ is the mean value, $\mathrm{A}$ is the amplitude of variation, $\omega$ is the frequency, $\theta$ is the phase.

The product of two sinusoidal curves with the same frequency can be derived as follows:

$$
\begin{aligned}
& f_{1}(t) f_{2}(t)=\left[\overline{\mathrm{M}}_{1}+\mathrm{A}_{1} \cdot \sin \left(\omega t+\theta_{1}\right)\right]\left[\overline{\mathrm{M}}_{2}+\mathrm{A}_{2} \cdot \sin \left(\omega t+\theta_{2}\right)\right] \\
& =\overline{\mathrm{M}}_{1} \overline{\mathrm{M}}_{2}+\mathrm{A}_{1} \mathrm{~A}_{2} \cos \left(\theta_{1}-\theta_{2}\right) \\
& +\sqrt{\left(\overline{\mathrm{M}}_{1} \mathrm{~A}_{2} \cos \theta_{2}+\overline{\mathrm{M}}_{2} \mathrm{~A}_{1} \cos \theta_{1}\right)^{2}+\left(\overline{\mathrm{M}}_{1} \mathrm{~A}_{2} \sin \theta_{2}+\overline{\mathrm{M}}_{2} \mathrm{~A}_{1} \sin \theta_{1}\right)^{2}} \cdot \sin \left(\omega t+\arctan \frac{\overline{\mathrm{M}}_{1} \mathrm{~A}_{2} \sin \theta_{2}+\overline{\mathrm{M}}_{2} \mathrm{~A}_{1} \sin \theta_{1}}{\overline{\mathrm{M}}_{1} \mathrm{~A}_{2} \cos \theta_{2}+\overline{\mathrm{M}}_{2} \mathrm{~A}_{1} \cos \theta_{1}}\right. \\
& -\frac{\mathrm{A}_{1} \mathrm{~A}_{2}}{2} \cos \left(2 \omega t+\theta_{1}+\theta_{2}\right)
\end{aligned}
$$

In Equation (35), there are three individual parts: mean value, sinusoidal fluctuation $(\omega)$, and sinusoidal fluctuation ( $2 \omega)$. Obviously, if an absolute operation is imposed on the fluctuation parts in Equation (35), sharp corners will be produced in the resultant curves. However, whether these sharp corners could be notable in the final result of $T \gamma$ depends on the combination of the contact parameters in specific cases, which is a complicated mathematical issue. The mean value of the contact responses, the fluctuation amplitude, and the phase between contact parameters can all influence the final results. Figure 4 shows the phase relations of contact responses excited by track irregularity and initial OOR respectively, which presents significant difference between each other. This could be one of the reasons why the sharp corners appear obviously in the curves of global methods for the case of initial OOR, while not for the case of track irregularity. Unfortunately it is not very clear as for why and how the phase relation of contact responses are formed for different scenarios.

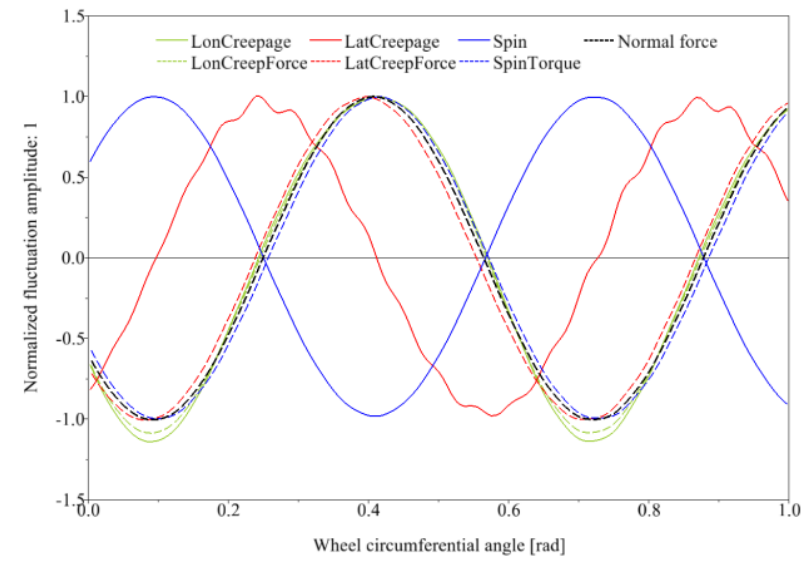

(a) Excited by vertical track irregularity

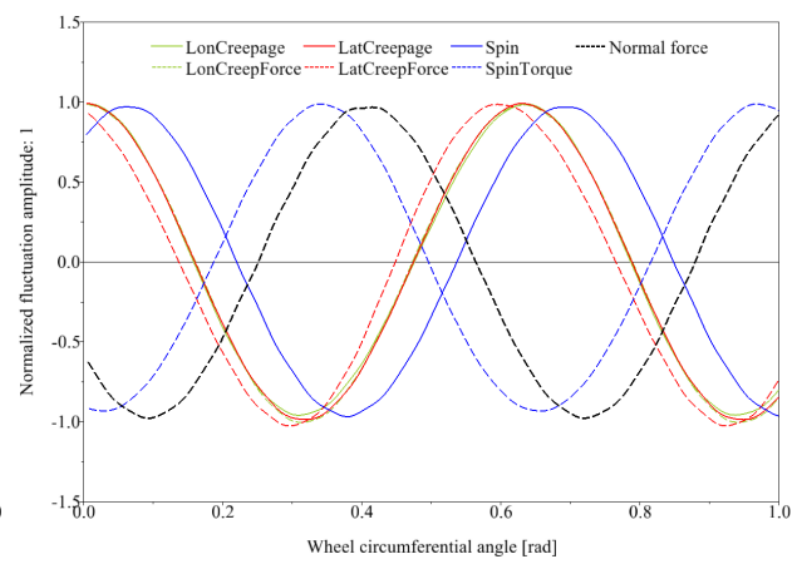

(b) Excited by initial OOR

Figure 4 Phase difference between contact responses: (a) Excited by vertical track irregularity, (b) Excited by initial OOR, with same excitating frequency and amplitude 


\subsection{Under random excitation}

Based on the understanding obtained from the case of harmonic excitation, 3 scenarios of random excitations are further investigated: equal-energy-distributed white noise, random track irregularity, and measured polygonal wear.

\subsubsection{Equal-energy-distributed white noise}

An important aspect in prediction of railway wheel polygonization is to identify the predominant resonant frequencies of the instantaneous wear in the vehicle/track dynamic system. White noise is a suitable excitation for this task. While it is worth noting that an equal-energy-distributed white noise is more preferable than white noise with a flat-PSD (power spectrum density with constant value). This is because that, given the same amplitude, excitation with higher frequency tends to produce higher responses than that with lower frequency due to more energy that higher excitations possess, which could interfere the observation of resonant frequencies. Consequently the energy should be equally distributed in white noise, which can be guaranteed by Equation (36).

$$
P(f) \times f^{2}=\text { constant }
$$

Where: $f$ is the distance frequency, $P(f)$ is the PSD amplitude at frequency f. Assuming: $P(1) \times 1^{2}=1$, Equation (36) can be written as:

$$
P(f)=1 / f^{2}
$$

Then an equal-energy-distributed white noise in distance domain can be generated by $P(f)$, but should be scaled to adapt for a reasonable track irregularity. In this case, a coefficient of 1e-10 is used. The resulting vertical track irregularity is shown in Figure 5. Note that the signal shown has been transformed to the time and frequency domain for the speed of $70 \mathrm{~km} / \mathrm{h}$. With this kind of vertical track irregularity as the excitation, the simulation results are shown in Figure 6.

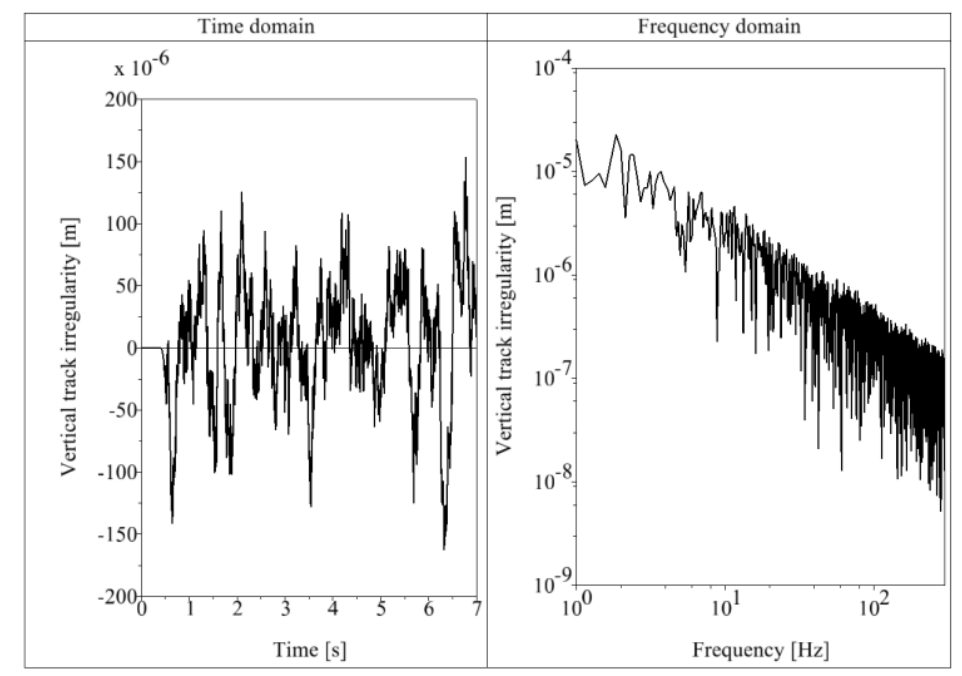

Figure 5 Vertical track irregularity generated by white noise with equally-distributed energy 


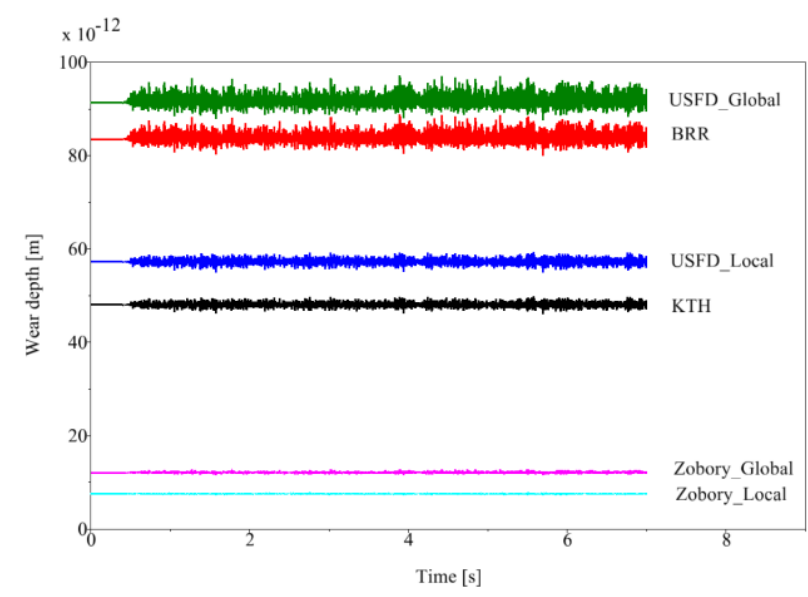

(a) Time history

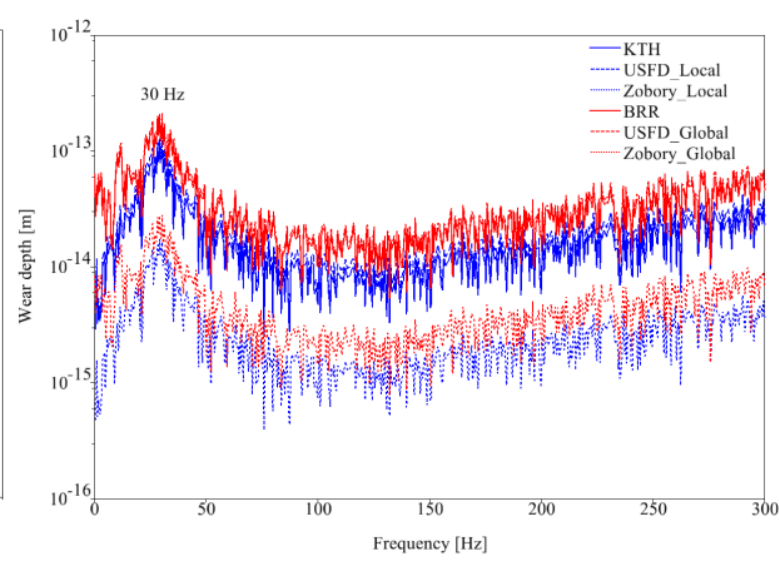

(b) Frequency spectrum

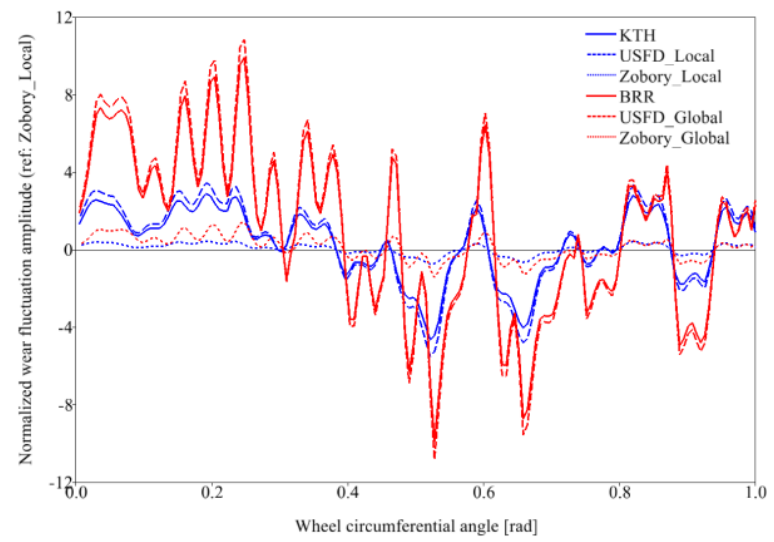

(c) Wear fluctuation phase and normalized wear fluctuation amplitude (ref: Zobory_Local)

Figure 6 Comparison of instantaneous wear depths among the wear models under excitation of equal-energydistributed white noise: (a) Time history, (b) Frequency spectrum, (c) Wear fluctuation phase and normalized wear fluctuation amplitude (ref: Zobory_Local)

As can be seen from Figure 6 (b), a resonant frequency of around $30 \mathrm{~Hz}$ in the instantaneous wear depth is distinguished by all the wear models. This frequency is a vertical modal frequency of the sleeper $\&$ wheelset, which is the easiest mode to be excited effectively in this case. By further checking Figure 6 (c), there are also some sharp corners appearing in the curves of global methods, which is similar to Figure 3 (c). On the contrary, all the curves from local methods look reasonable.

\subsubsection{Standard track irregularity}

A standard track irregularity FRA 6 (the $6^{\text {th }}$ class track defined by Federal Railroad Administration of US) [24] is adopted to investigate the responses of wear modes under excitation of a realistic random track irregularity. In reality the main energy of the random track irregularity is normally distributed in the range of long wavelengths corresponding to the range of low time frequencies (below $12 \mathrm{~Hz}$ at speed of $70 \mathrm{~km} / \mathrm{h}$ in this case). The simulation results are presented in Figure 7. 


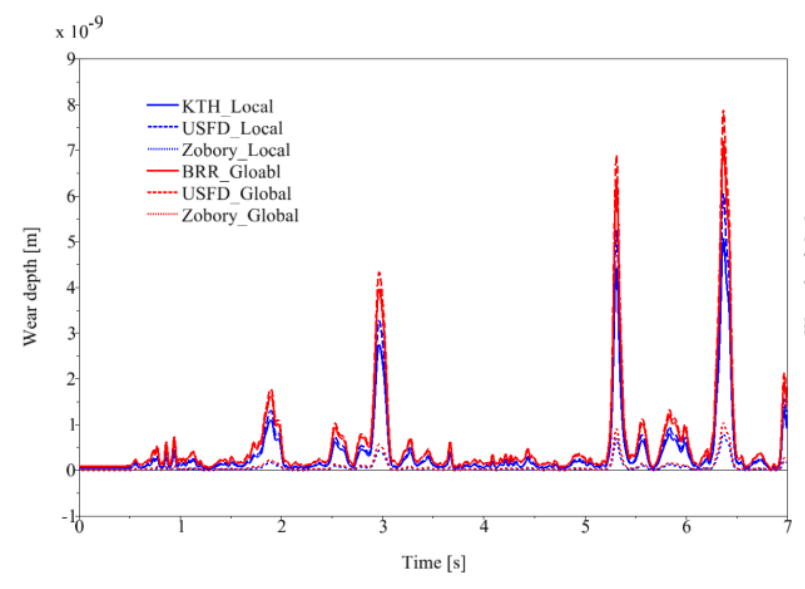

(a) Time history

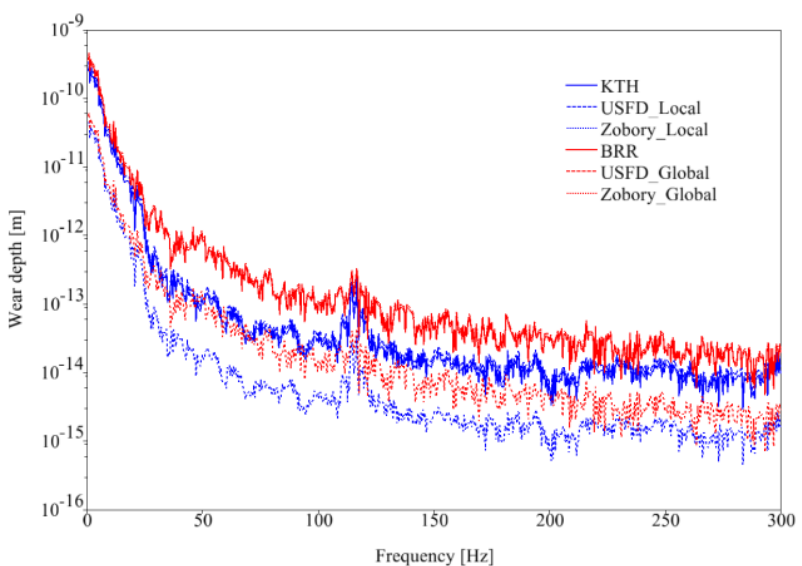

(b) Frequency spectrum

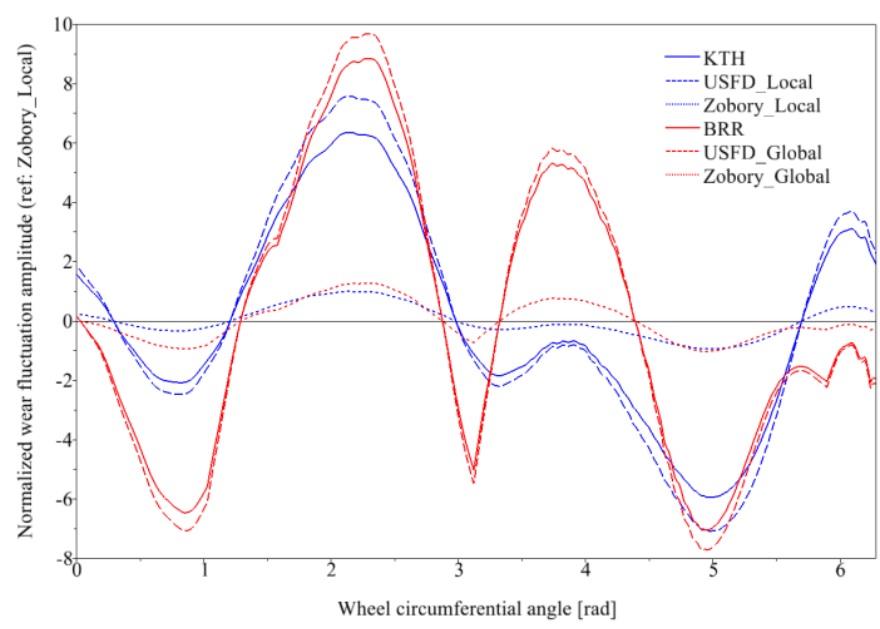

(c) Wear fluctuation phase and normalized wear fluctuation amplitude (ref: Zobory_Local)

Figure 7 Comparison of instantaneous wear depths among the wear models under excitation of random track irregularity (AAR6): (a) Time history, (b) Frequency spectrum, (c) Wear fluctuation phase and normalized wear fluctuation amplitude (ref: Zobory_Local)

As can be seen from Figure 7 (a) and (b), the calculated instantaneous wear depths are still comparable between each wear model. The energy concentrated upon the lower frequencies are correctly reflected by all the wear models. Note that the wear excited by a random track irregularity is much larger than other cases. Although some abrupt wear depths can be found in the time history, it will not produce unrealistic results for the final polygonal wear, because for a random case, a long enough sample must be adopted and then averaged for many revolutions, so abrupt peaks will be eliminated. When looking at Figure 7 (c), some sharp corners are still found in the curves of global methods.

\subsubsection{Measured polygonal wear}

In reality, the railway wheel polygonalization is gradually formed not only by the track excitation but also due to the polygonal wear itself. The measured polygonal wear are adopted to investigate the responses of the wear models under the excitation of realistic initial polygonal wear. Figure 8 shows the measured polygonal wear of 8 wheels of the locomotive investigated in this paper, with the average value indicated by a red line. More details with respect to the statistical measurement can be found in [21]. This measured polygonal wear is assigned to each wheel respectively. The simulation results are showed in Figure 9. 

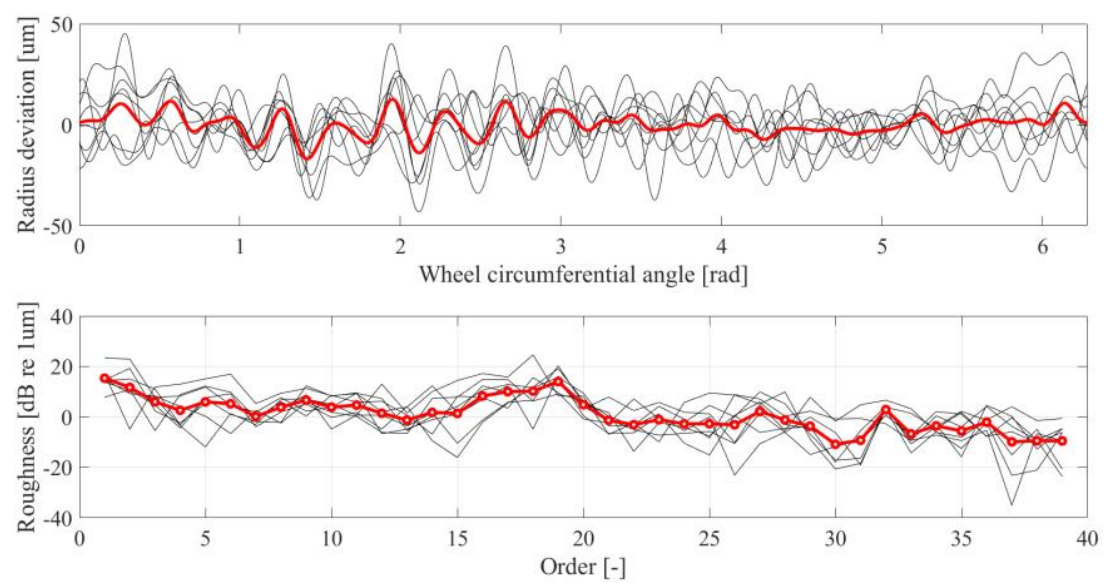

Figure 8 Measured polygonal wear of a locomotive: Black lines indicating 8 wheels, the red line indicating the averaged value

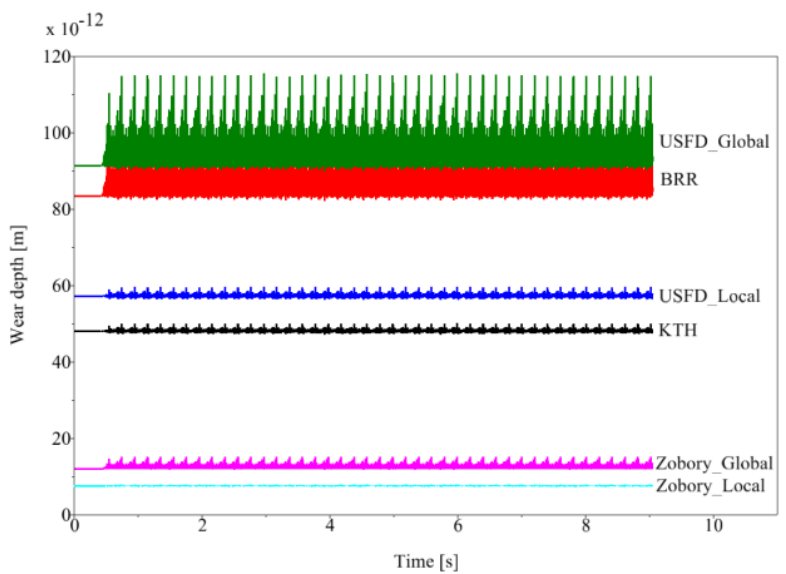

(a) Time history

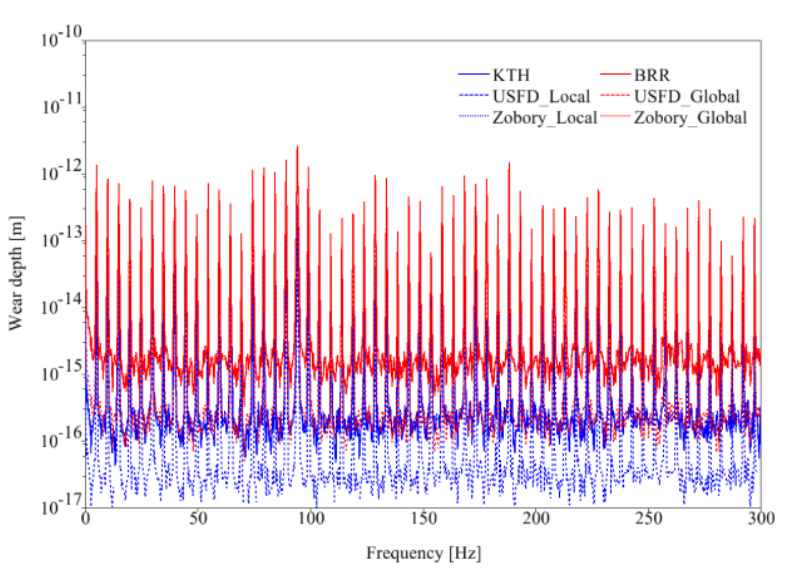

(b) Frequency spectrum

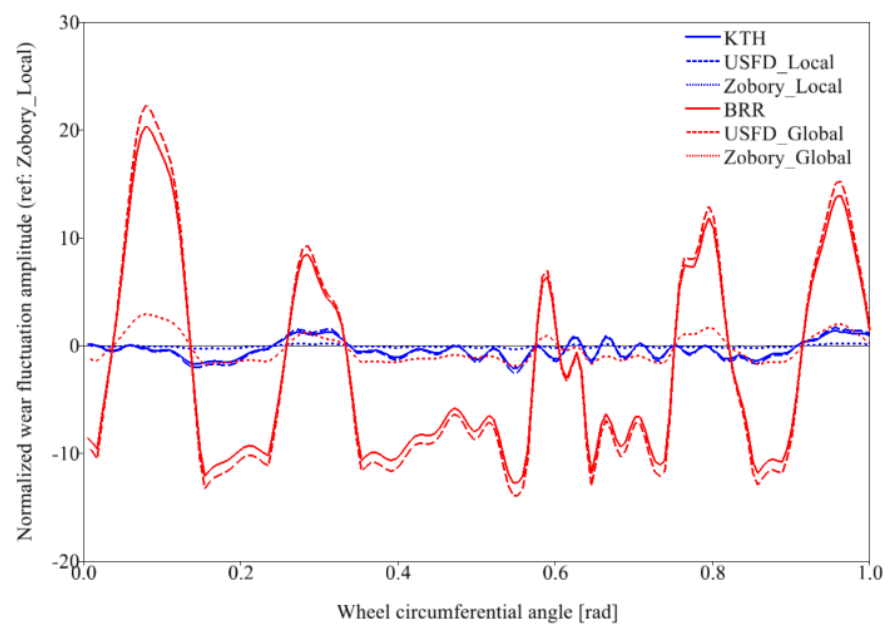

(c) Wear fluctuation phase and normalized wear fluctuation amplitude (ref: Zobory_Local)

Figure 9 Comparison of instantaneous wear depths among the wear models under excitation of Measured initial wheel polygonization: (a) Time history, (b) Frequency spectrum, (c) Wear fluctuation phase and normalized wear fluctuation amplitude (ref: Zobory_Local)

A dominant feature in Figure 9 (b) is the notable multiple frequency components. The interval of these multiple frequency components is exactly the rolling frequency of the wheels, which is around $5 \mathrm{~Hz}$ in this case. This is 
due to the cyclic excitation by the polygonal wear itself. Despite this, all wear models present a similar trend of responses only except that some sharp corners persist in the curves of global methods.

\subsection{Evolution of polygonal wear}

Prediction of railway wheel polygonalization is a complicated topic on its own, which will not be elaborated in detail in this paper. This section is only to present the evolved polygonal wear around the wheel circumference under two tentative (proposed?) circumstances: sinusoidal excitation and white noise, with concentration on the comparison between each wear model. For consistency, two scenarios specified in Section 3.1.1 (Vertical sinusoidal track irregularity) and Section 3.2.1 (Equal-energy-distributed white noise) are adopted as the tentative (proposed?) scenarios here, and all the model parameters as well as the original wear coefficients are set the same as the above sections. A developed program [17] is applied to predict the wheel polygonalization. The number of iterations is set to 10 with an amplification coefficient of $1 \mathrm{e} 6$ for each iteration. So the corresponding total running distance is $39,250 \mathrm{~km}$. The final evolution of the polygonal wear using different wear models under two tentative scenarios is presented in Figure 10 and Figure 11 respectively.

\subsubsection{Under excitation of sinusoidal vertical track irregularity}

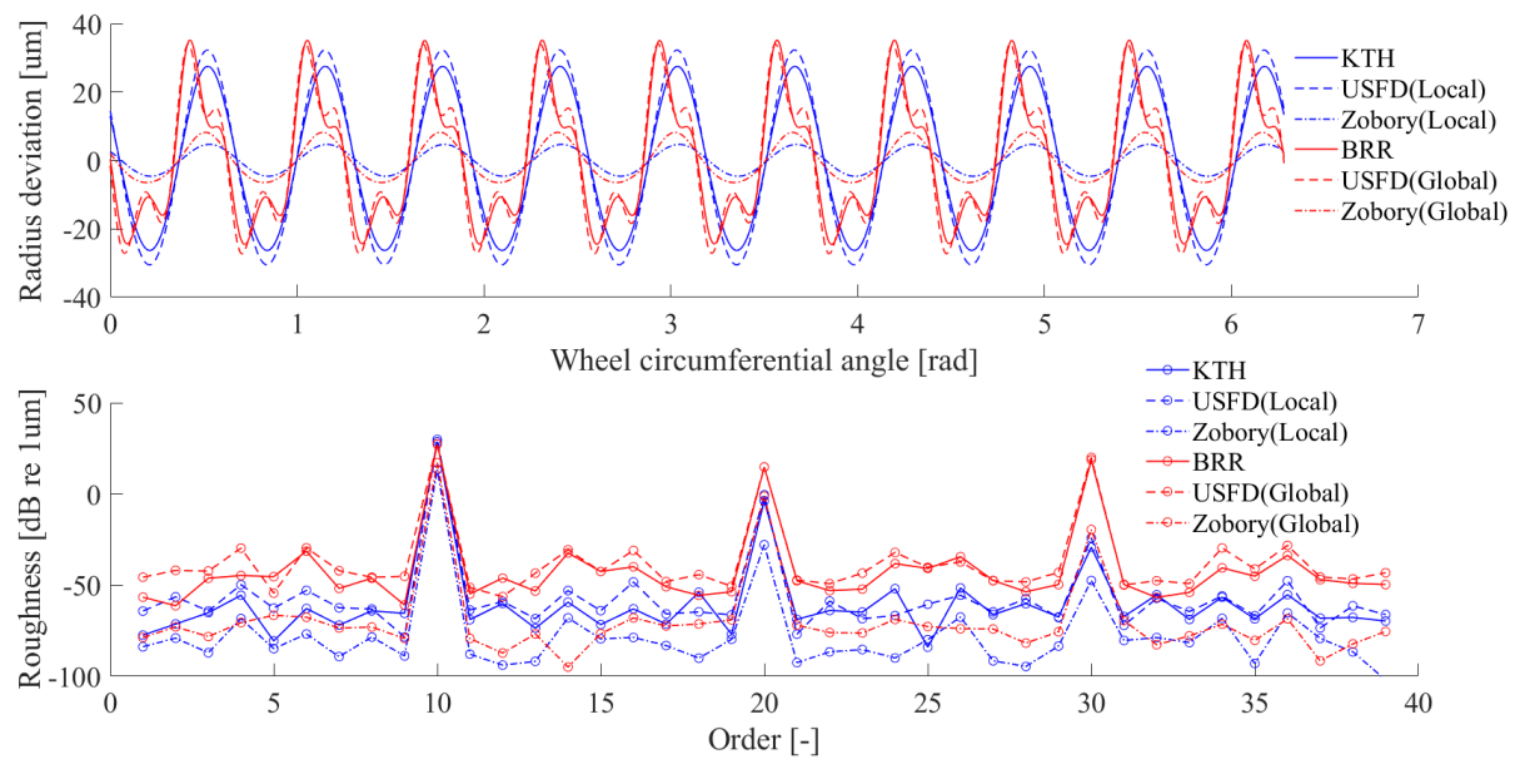

Figure 10 Evolution of polygonal wear under excitation of sinusoidal vertical track irregularity

As can be seen from Figure 10, all the wear models indicate the dominantly developed order of 10 which corresponds to the excitation frequency of $50 \mathrm{~Hz}$ at speed of $70 \mathrm{~km} / \mathrm{h}$. The evolved radius deviation as well as the growth rate of the wheel roughness depend on the original wear coefficients used in each wear model. With a close look, all the wear models of local methods present a good consistency with respect to the phase as well as the gradually attenuated multiple frequency components. However, in terms of the global methods the situation is relatively complicated. Remember that the excitation of vertical sinusoidal track irregularity itself will not produce obvious sharp corners in the global instantaneous wear (see Figure 2 (c)), but the newly developed polygonal wear during iterations will (see Figure 3 (c)). Meanwhile, the evolution program will have to smooth the polygonal wear curves between iterations to guarantee a stable successive simulation. When the newly developed polygonal wear becomes large enough, some sharp corners will be generated but will be smoothed as well. If the evolved radius deviation is big (BRR and USFD (Global)), the smoothed sharp corners can be observed easily, while if not 
(Zobory (Global)), the smoothed sharp corners cannot be observed easily. At the same time, for the largely evolved radius deviation (BRR and USFD (Global)), some multiple frequency components not gradually attenuated can be observed due to the smoothed curves of sharp corners.

In addition, another aspect of interest is the proportional relation of the evolved wheel roughness between each wear model. The ratio of the final wheel roughness of the $10^{\text {th }}$ order between each wear model is compared with that of the mean value of the instantaneous wear depth for the first run, and is listed in the following Table 5. As can be seen from Table 5, the proportional relationship among the wear models except BRR and USFD (Global) persists well generally. The change of BRR and USFD (Global) in the proportional relationship is due to the smoothed curves of sharp corners which has been explained above. The slightly change of the local wear models in the proportional relationship is attributable to the iterations in which newly evolved polygonal wear are always introduced for the next run. Remember that the ratio of the mean value of the instantaneous wear depth between each wear model is determined by the equivalent coefficients (see Section 3.1.1). This is to say the equivalent coefficients can also be employed to estimate the proportional relationship of the final evolved wheel roughness among the wear models.

Table 5 A comparison between the ratio of the mean value and the ratio of fluctuation amplitude

\begin{tabular}{llllllll}
\hline \multirow{2}{*}{ Model } & \multirow{2}{*}{ BRR } & $\begin{array}{l}\text { USFD } \\
\text { Global }\end{array}$ & $\begin{array}{l}\text { Zobory } \\
\text { Global }\end{array}$ & KTH & $\begin{array}{l}\text { USFD } \\
\text { Local }\end{array}$ & $\begin{array}{l}\text { Zobory } \\
\text { Local }\end{array}$ \\
\hline \multirow{2}{*}{ Ratio } & Mean value (first run) & 11.04 & 12.1 & 1.6 & 6.36 & 7.58 & 1 \\
& Wheel roughness (final result) & 5 & 5.1 & 1.56 & 5.79 & 6.75 & 1 \\
\hline
\end{tabular}

* The Zobory wear model locally is the reference for the ratio calculation

\subsubsection{Under excitation of equal-energy-distributed white noise}

The results of global methods and local methods are displayed separately in Figure 11 to facilitate the observation. First of all, the $6^{\text {th }}$ order which should develop dominantly according to the main resonant frequency of around 30 $\mathrm{Hz}$ at speed of $70 \mathrm{~km} / \mathrm{h}$ (see Figure 6 (b)), is successfully distinguished by all the wear models. Having a close look at Figure 11 (a), all wear models of local methods present a very good consistency in simulating the polygonal wear around the wheel circumference after a long distance of running under excitation with white noise. However on the contrary, the global methods do not show perfect consistency between each other, as shown in Figure 11 (b). This is also due to the smoothing operation on the curves of sharp corners, by which some artificial errors may have been introduced. 


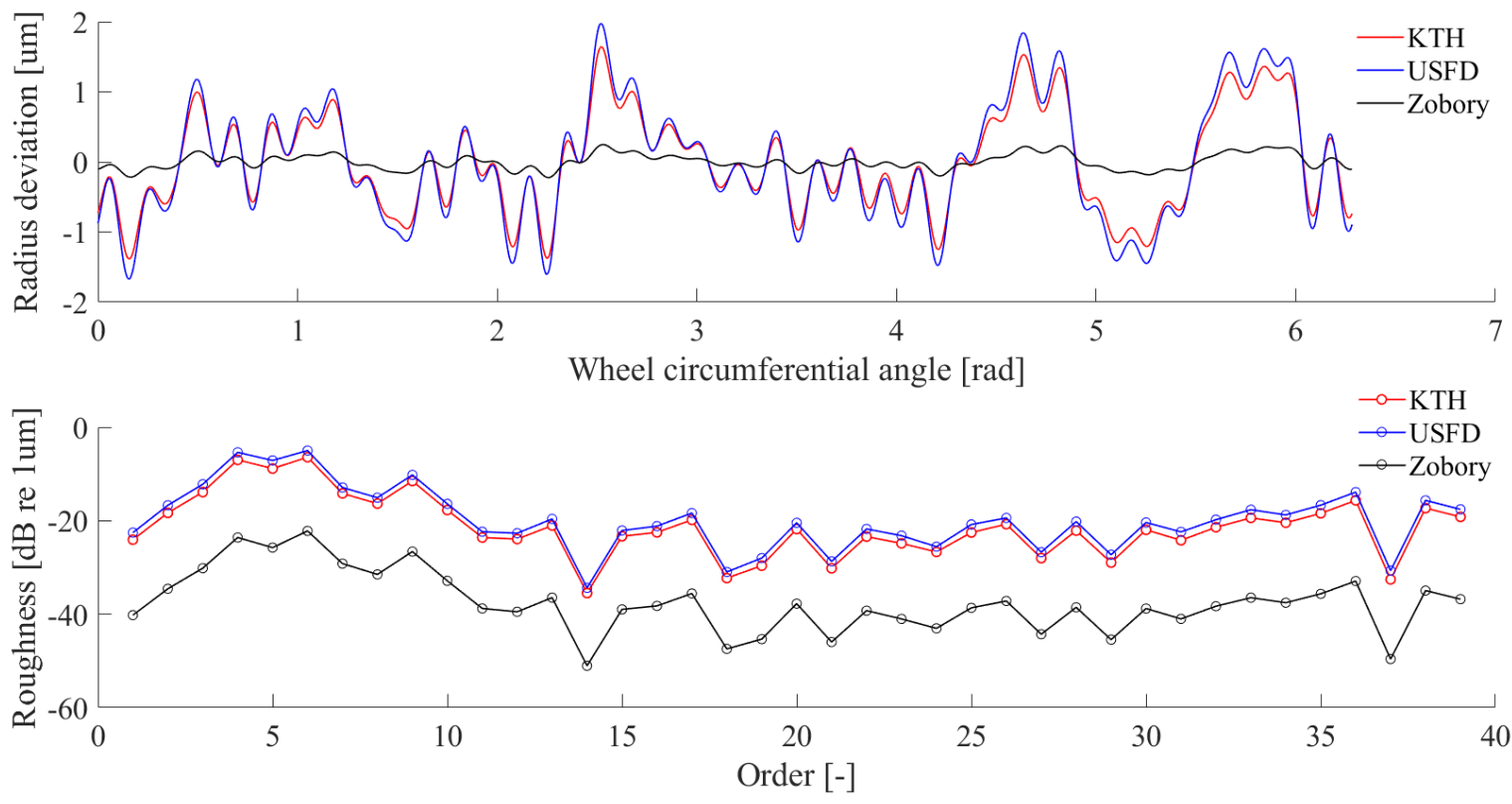

(a) Local methods

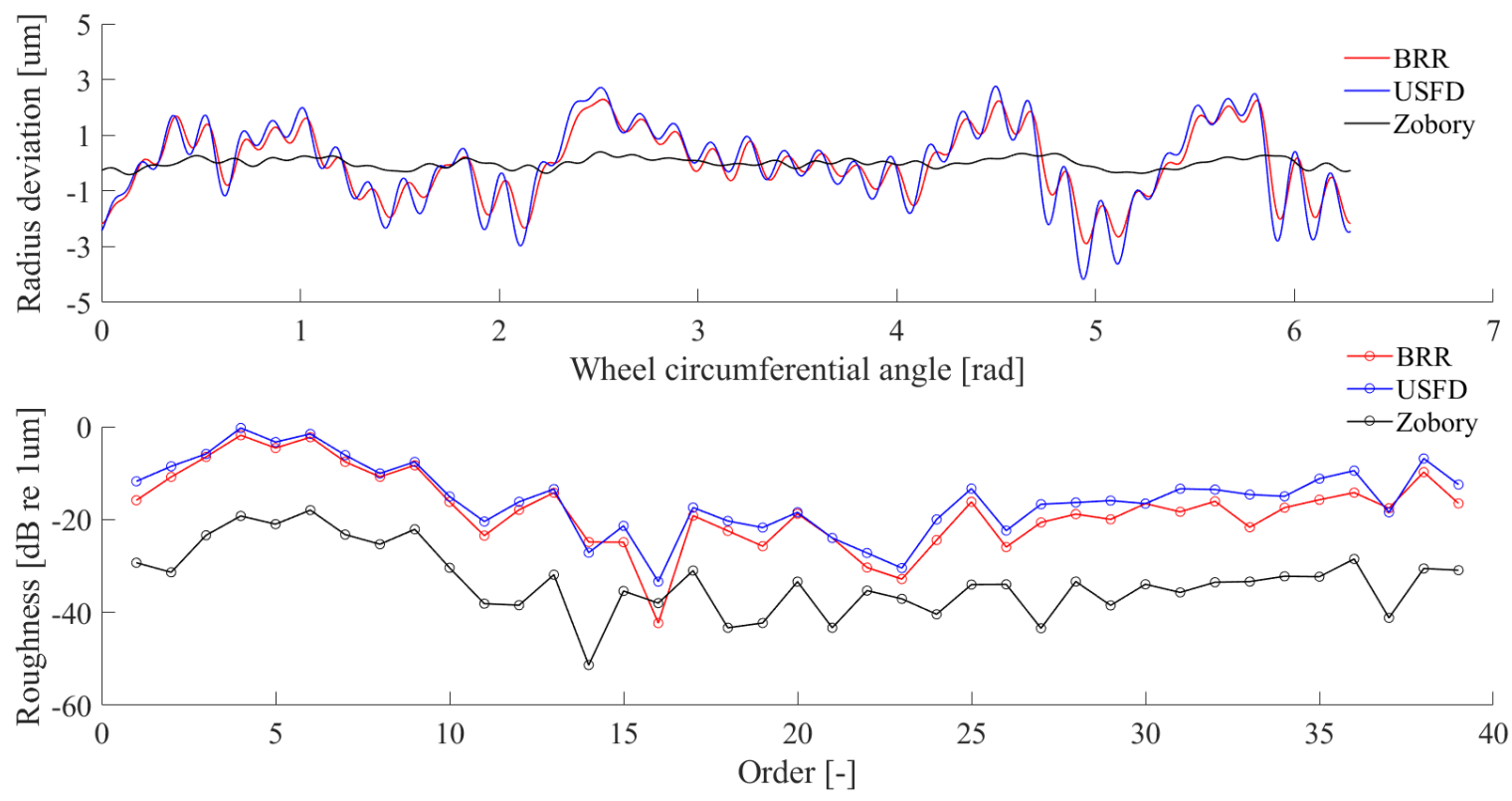

(b) Global methods

Figure 11 Evolution of polygonal wear under excitation of equal-energy-distributed white noise: (a) Local methods;

(b) Global methods

\section{Discussion}

\subsection{About the sharp corners}

As can be seen in Section 0, some sharp corners can always be found in the instantaneous wear depth calculated by global methods for most scenarios. It is due to the absolute operation for the global WI $T \gamma$, which has been explained in Section 3.1.2. This situation will not happen for local methods which avoid absolute operation. Although there are still some square operation for local WI $\tau(x, y) \xi(x, y)$ (see Equation (2)) that may produce sharp corners for one element wear, the average of the meshed wear distribution is always able to smooth some potential sharp corners. Although the sharp corners generated by global methods are possible to be smoothened 
with some solutions, it takes a risk of causing artificial errors in the results. Therefore, it is naturally believed that the global method is not suitable for calculating the instantaneous wear, although it could reduce some computing time, and the local method is recommended.

\subsection{About the assumptions}

When studying wear in the wheel transversal profile, the wheel circumferential profile was always assumed unaltered [3, 9, 12, 14, 25-30]. Vice versa, the wheel transversal profile is assumed not changed in this paper so as to concentrate on the wheel polygonalization, which is also supported by the statistical measurement [21, 22]. Based on this assumption, the instantaneous wear of one contact patch is further assumed to be equally distributed in the wheel transversal profile. That is to say the instantaneous wear is averaged to get the final wear depth. However, an alternative handling of the wear distribution is adopting the maximum wear value of the transversal wear curve as the final result, for example see [18]. A comparative study has been conducted to check the difference between these two methods. It turns out that the shape of the calculated circumferential wear is rather similar for most scenarios, but with a difference being the fluctuation amplitude which is the key parameter for the growth rate. Even though, it is not believed to be a problem because the wear coefficient that determines the growth rate of wheel polygonalization should always be adjusted to a real case after all. Actually the wear coefficients originally developed for predicting the wheel transversal profile might not be suitable for prediction of railway wheel polygonalization as the focus interested is rather different from each other. As a result, the handling of the wear distribution may not be a critical issue, but the average operation is more preferable.

In addition, the contact model is assumed to use Hertz theory for normal contact and FASTSIM for tangential contact. This is the most common combination adopted for wheel/rail contact calculation. If other contact models are used, the conversion of the wear models might have to be adjusted accordingly, and the derived equations might not be very concise to facilitate the comparison between each wear model. However, it is still believed that the derived uniform wear functions together with the equivalent coefficients in this paper can reflect the basic nature and the proportional relation among the wear models in a general sense.

\subsection{About the local WI}

The local WI $\tau(x, y) \xi(x, y)$ of one grid element is defined as the product of the resultant tangential stress and the resultant local creepage. From a mathematical view, this is a product of two vectors that have the same direction. Calculation is less clear as two different methods are available in the academic community, which are listed below:

$$
\begin{gathered}
\text { Method 1: } \tau(x, y) \xi(x, y)=\sqrt{\tau_{x}^{2}(x, y)+\tau_{y}^{2}(x, y)} \cdot \sqrt{\xi_{x}^{2}(x, y)+\xi_{y}^{2}(x, y)} \\
\text { Method 2: } \tau(x, y) \xi(x, y)=\tau_{x}(x, y) \xi_{x}(x, y)+\tau_{y}(x, y) \xi_{y}(x, y)
\end{gathered}
$$

In the original paper written by Professor Zobory [3], although the expression of $\tau(x, y) \xi(x, y)$ is given by method 2, the calculation of it is actually according to method 1, which can be seen in the Equation (20) in reference [3]. In addition, the Archard wear model upon which the KTH wear model is based essentially uses method 1 to calculate its original global WI $N d$ which can also be converted to $\tau(x, y) \xi(x, y)$ (see Section 2.3.2). As the local WI $\tau(x, y) \xi(x, y)$ represents the frictional work which is not a vector but a scalar, it is believed that method 1 is the preferable one. Obviously, the result calculated by method 2 will be slightly smaller than that by method 
1. In addition, as $\tau(x, y)$ and $\xi(x, y)$ share the same direction, an absolute operation in method 2 is actually not necessary.

\section{Conclusion}

To sum up, although the wear models being investigated in this paper are different from each other in their original definition, they actually share the same nature in calculating the instantaneous wear depth. In other words, the WI is exactly the same among the wear models, although the expression of WI is different for global and local methods. A uniform expression can be analytically derived for all the wear models, with the equivalent coefficients conveniently indicating their proportional relationship in a quantitative sense. Besides, all the wear models present a similar ability to reflect the fluctuation of the wheel polygonal wear in a general sense. Specifically, the fluctuation frequency and fluctuation phase of the polygonal wear are obtained with almost the same results using different wear models. While the mean value and the fluctuation amplitude of the polygonal wear are both determined by the original wear coefficients used. Besides, the global method is believed not suitable for calculating the polygonal wear of railway wheels, and the local method is recommended.

\section{References}

[1] P. Bolton, P. Clayton, Rolling-sliding wear damage in rail and tyre steels, Wear, 93 (1984) 145-165.

[2] T.G. Pearce, N.D. Sherratt, Prediction of wheel profile wear, Wear, 144 (1991) 343-351.

[3] I. Zobory, Prediction of Wheel/Rail Profile Wear, Vehicle System Dynamics, 28 (1997) 221-259.

[4] J. Neilsen, Evolution of rail corrugation predicted with a non-linear wear model, Journal of Sound and Vibration, 227 (1999) 915-933.

[5] T. Jendel, M. Berg, Prediction of Wheel Profile Wear, Vehicle System Dynamics, 37 (2002) 502-513.

[6] R. Lewis, R.S. Dwyer-Joyce, Wear mechanisms and transitions in railway wheel steels, Proceedings of the Institution of Mechanical Engineers, Part J: Journal of Engineering Tribology, 218 (2004) 467-478.

[7] R. Enblom, On Simulation of Uniform Wear and Profile Evolution in the Wheel-Rail Contact, Department of Aeronautical and Vehicle Engineering, Royal Institute of Technology (KTH), 2006.

[8] T.T. Vuong, P.A. Meehan, D.T. Eadie, K. Oldknow, D. Elvidge, P.A. Bellette, W.J. Daniel, Investigation of a transitional wear model for wear and wear-type rail corrugation prediction, Wear, 271 (2011) 287-298.

[9] J. De Arizon, O. Verlinden, P. Dehombreux, Prediction of wheel wear in urban railway transport: comparison of existing models, Vehicle System Dynamics, 45 (2007) 849-874.

[10] J.F. Archard, Contact and Rubbing of Flat Surfaces, Journal of Applied Physics, 24 (1953) 981.

[11] R. Enblom, M. Berg, Simulation of railway wheel profile development due to wear-influence of disc braking and contact environment, Wear, 258 (2005) 1055-1063.

[12] J. Pombo, J. Ambrósio, M. Pereira, R. Lewis, R. Dwyer-Joyce, C. Ariaudo, N. Kuka, Development of a wear prediction tool for steel railway wheels using three alternative wear functions, Wear, 271 (2011) 238-245.

[13] J. Ding, F. Li, Y. Huang, Analysis of the wheel wear model based on the creep mechanism, Zhongguo Tiedao Kexue, 31 (2010) 66-72.

[14] F. Braghin, R. Lewis, R.S. Dwyer-Joyce, S. Bruni, A mathematical model to predict railway wheel profile evolution due to wear, Wear, 261 (2006) 1253-1264.

[15] A. Johansson, Out-of-Round Railway Wheels - Causes and Consequences, Department of Applied Mechanics, Chalmers University of Technology, Gotherberg, Sweden, 2005.

[16] X. Wu, S. Rakheja, W. Cai, M. Chi, A. Ahmed, S. Qu, A study of formation of high order wheel polygonalization, Wear, 424 (2019) 1-14.

[17] B. Peng, S. Iwnicki, P. Shackleton, Y. Zhao, D. Cui, A practical method for simulating the evolution of railway wheel polygonalization, 25th international symposium on dynamics of vehicles on roads and tracks, Rockhampton, Australia, 2017, pp. 753-758.

[18] A. Johansson, C. Andersson, Out-of-round railway wheels-a study of wheel polygonalization through simulation of three-dimensional wheel-rail interaction and wear, Vehicle System Dynamics, 43 (2005) 539-559.

[19] B. Morys, Enlargement of out-of-round wheel profiles on high speed trains, Journal of Sound and Vibration, 227 (1999) 965-978. 
[20] G. Tao, Investigation into the formation mechanism of the polygonal wear of HXD electric locomotive wheels, Southwest Jiaotong University, 2018.

[21] G. Tao, L. Wang, Z. Wen, Q. Guan, X. Jin, Measurement and assessment of out-of-round electric locomotive wheels, Proceedings of the Institution of Mechanical Engineers, Part F: Journal of Rail and Rapid Transit, 232 (2018) 275-287.

[22] A. Johansson, Out-of-round railway wheels - assessment of wheel tread irregularities in train traffic, Journal of Sound and Vibration, 293 (2006) 795-806.

[23] J.J. Kalker, A Fast Algorithm for the Simplified Theory of Rolling Contact, Vehicle System Dynamics, 11 (1982) 1-13.

[24] V.K. Garg, R.V. Dukkipati, Dynamics of railway vehicle systems, Academic Press, Toronto, Ont, 1984.

[25] G.-q. Tao, X. Du, H.-j. Zhang, Z.-f. Wen, X.-s. Jin, D.-b. Cui, Development and validation of a model for predicting wheel wear in high-speed trains, Journal of Zhejiang University-SCIENCE A, 18 (2017) 603-616.

[26] J. Ding, F. Li, Y. Huang, S. Sun, L. Zhang, Application of the semi-Hertzian method to the prediction of wheel wear in heavy haul freight car, Wear, 314 (2014) 104-110.

[27] R. Enblom, S. Stichel, t. Skolan för, Kth, Spårfordon, f. Farkost och, Industrial implementation of novel procedures for the prediction of railway wheel surface deterioration, Wear, 271 (2011) 203-209.

[28] A. Bevan, P. Allen, Application of a wear prediction method to the analysis of a new UK wheel profile, 2006. [29] U. Olofsson, T. Telliskivi, Wear, plastic deformation and friction of two rail steels - a full-scale test and a laboratory study, Wear, 254 (2003) 80-93.

[30] T. Jendel, Prediction of wheel profile wear-comparisons with field measurements, Wear, 253 (2002) 89-99. 


\section{Notation}

\begin{tabular}{|c|c|}
\hline$T_{x}$ & longitudinal tangential force $[\mathrm{N}]$ \\
\hline$T_{y}$ & lateral tangential force $[\mathrm{N}]$ \\
\hline$M$ & spin torque $[\mathrm{Nm}]$ \\
\hline$\gamma_{x}$ & global longitudinal creepage global [-] \\
\hline$\gamma_{y}$ & global lateral creepage [-] \\
\hline$\phi$ & $\operatorname{spin}[1 / \mathrm{m}]$ \\
\hline$\tau$ & resultant tangential stress $\left[\mathrm{N} / \mathrm{m}^{2}\right]$ \\
\hline$\tau_{x}$ & longitudinal tangential stress $\left[\mathrm{N} / \mathrm{m}^{2}\right]$ \\
\hline$\tau_{y}$ & lateral tangential stress $\left[\mathrm{N} / \mathrm{m}^{2}\right]$ \\
\hline$\xi$ & local creepage (resultant) [-] \\
\hline$\xi_{x}$ & local longitudinal creepage $[-]$ \\
\hline$\xi_{y}$ & local lateral creepage [-] \\
\hline$W_{\text {(wear model) }}$ & wear rate [depending on wear model] \\
\hline$K_{\text {(wear model) }}$ & original wear coefficient [depending on wear model] \\
\hline$K_{(\text {wear model) }}^{*}$ & equivalent wear coefficient for global methods $[\mathrm{m} / \mathrm{N}]$ \\
\hline$K_{(\text {wear model) }}^{\#}$ & equivalent wear coefficient for local methods $\left[\mathrm{m}^{3} / \mathrm{N}\right]$ \\
\hline$D$ & wheel diameter $[\mathrm{m}]$ \\
\hline$A$ & contact area $\left[\mathrm{m}^{2}\right]$ \\
\hline$\dot{E}$ & stochastic energy flow density $\left[\mathrm{Nm} / \mathrm{sm}^{2}\right]$ \\
\hline$H_{\text {(wear model) }}$ & global wear depth [m] \\
\hline$h_{\text {(wear model) }}$ & local wear depth $[\mathrm{m}]$ \\
\hline$a$ & longitudinal semi-axis [m] \\
\hline$b$ & lateral semi-axis $[\mathrm{m}]$ \\
\hline$N$ & normal force $[\mathrm{N}]$ \\
\hline$d$ & sliding distance $[\mathrm{m}]$ \\
\hline $\mathrm{H}$ & hardness of material [pa] \\
\hline$p_{z}$ & normal pressure $\left[\mathrm{N} / \mathrm{m}^{2}\right]$ \\
\hline $\mathrm{P}_{\mathrm{z}}$ & mean normal pressure $\left[\mathrm{N} / \mathrm{m}^{2}\right]$ \\
\hline$\Delta d$ & local sliding distance $[\mathrm{m}]$ \\
\hline$\mu$ & friction coefficient $[-]$ \\
\hline$s$ & resultant sliding velocity in grid element $[\mathrm{m} / \mathrm{s}]$ \\
\hline$V$ & vehicle speed $[\mathrm{m} / \mathrm{s}]$ \\
\hline$\Delta x$ & longitudinal length of one grid element [m] \\
\hline$m$ & longitudinal meshing number [-] \\
\hline$\rho$ & material density $\left[\mathrm{kg} / \mathrm{m}^{3}\right]$ \\
\hline$v_{x}$ & global longitudinal sliding velocity $[\mathrm{m} / \mathrm{s}]$ \\
\hline$v_{y}$ & global lateral sliding velocity $[\mathrm{m} / \mathrm{s}]$ \\
\hline$\omega$ & angular sliding velocity $[\mathrm{rad} / \mathrm{s}]$ \\
\hline$d t$ & global passing time through one contact patch $[\mathrm{s}]$ \\
\hline$\Delta t$ & local passing time through one grid element $[\mathrm{s}]$ \\
\hline
\end{tabular}

